Discussion Paper No. 909

\title{
SOCIAL LEARNING AND DELAY IN A DYNAMIC MODEL OF PRICE COMPETITION
}

\author{
Masaki Aoyagi \\ Manaswini Bhalla \\ Hikmet Gunay
}

July 2014

The Institute of Social and Economic Research Osaka University

6-1 Mihogaoka, Ibaraki, Osaka 567-0047, Japan 


\title{
Social Learning and Delay in a Dynamic Model of Price Competition
}

\author{
Masaki Aoyagi* Manaswini Bhalla ${ }^{\dagger}$ Hikmet Gunay ${ }^{\ddagger}$
}

July 18, 2014

\begin{abstract}
This paper studies dynamic price competition over two periods between two firms selling differentiated durable goods to two buyers who are privately informed about their types, but have valuations of the two goods dependent on the other buyer's type. The firms' pricing strategy in period 1 must take into account the buyers' incentive to wait and learn from the other buyer's decision. We construct an equilibrium based on the key observation that the expected price of either good in period 2 is the same as its price in period 1 on and off the path of play. The equilibrium is shown to be non-preemptive in the sense that even if either firm fails to make a sale in period 1, it still makes a sale with positive probability in period 2. A characterization of the equilibrium is given in terms of the probability of delay as a function of the degree of interdependence between the two buyers.

Key words: dynamic pricing, delay, social learning, duopoly, product differentiation, durable good, preemption, revenue management.

Journal of Economic Literature Classification Numbers: C72, D82.
\end{abstract}

\section{Introduction}

Consumer preferences are intrinsically interdependent in many durable goods markets. Consider, for example, potential consumers of a new model of an automobile. Purchase decisions of such a product are accompanied by careful examination of various information about it: Consumers collect information from a catalog and magazine articles as well as from their own experience of products from the same manufacturer. They then

\footnotetext{
*ISER, Osaka University (aoyagi@iser.osaka-u.ac.jp).

${ }^{\dagger}$ Economics and Social Science, Indian Institute of Management, Bangalore (manaswinib@iimb.ernet.in).

${ }^{\ddagger}$ Department of Economics, University of Manitoba (Hikmet.Gunay@umanitoba.ca).
} 
summarize such information to form an opinion as an estimate of his valuation of the product. However, the individual piece of information is arguably imperfect, and each consumer has a better estimate of his valuation when the opinions of all consumers are pooled. ${ }^{1}$ When direct and truthful communication of opinions is not feasible, hence, each consumer has an incentive to wait and see the decisions of other consumers to indirectly learn their opinions.

We are interested in the problem of intertemporal price competition between two firms selling differentiated durable goods to such interdependent consumers. In our model, two consumers each have private opinions about the relative superiority of the two goods, and buy a single unit of either good in one of the two periods in an irreversible manner. Hence, a consumer in period 1 must decide between buying today from either firm for the quoted price, and waiting until tomorrow. If he waits, he has better information about his valuation, but the price offer by each firm in period 2 is also contingent on the buyers' decision and can be high or low depending on whether its product was chosen in period 1. Each firm, on the other hand, needs to set its price taking into account the consumers' incentives to 'wait and see' as well as the pricing decision of the other firm. For example, by offering a discount in period 1, a firm may preempt the market by capturing one of the consumers and then be able to sell the good to the other consumer at a higher price in period 2. On the other hand, offering a discount may be detrimental to the profits if, for example, it leads to a more intense competition in period 2. Further, each firm needs to take into account the information flow generated by its pricing decision. This simple discussion already suggests the complexity of the strategic interaction between the consumers, between the firms, and between the firms and the consumers.

A more detailed description of our model is as follows: Two firms $A$ and $B$ sell durable goods $A$ and $B$, respectively, over two periods. There are two consumers $i=1,2$ each of whom is endowed with the private type $s_{i}$ which represents his opinion about the goods as described above. We assume that the type $s_{i}$ has a one-dimensional continuous

\footnotetext{
${ }^{1}$ A consumer's opinion hence may be a noisy signal about the true (subjective) value of the product, or may be his subjective valuation of it. In the former interpretation, the two opinions will be positively correlated if they are information about the same aspect of the product but can be independent if they are about different aspects of it (e.g., driving performance and fuel efficiency of an automobile). Valuations can be interdependent even when the opinions are subjective if, for example, consumers are concerned with how they are perceived by others. As will be seen, the present paper formulates interdependent valuations based on independent opinions. While independence is assumed mainly for simplicity, the same formulation is frequently used in the mechanism design literature. See for example Jehiel and Moldovanu (2001).
} 
distribution. We express the interdependence of their preferences by assuming that buyer $i$ 's valuation of either good is the weighted sum of his and the other buyer's types. We also assume that the consumers' preferences are idiosyncratic by supposing that the weight placed on the own opinion is larger than that placed on the other consumer's opinion. The types are ordered so that the higher the type $s_{i}$, the higher is buyer $i$ 's relative valuation of good $B .^{2}$ Each consumer demands at most one unit of either good and purchases the product at most once in one of the two periods. In period 1, the firms quote prices simultaneously, and the consumers make simultaneous decisions on whether to buy either good or wait until period 2. The public history comprises the prices and consumer decisions in period 1. Given the updated belief about the consumers' types, the firms in period 2 again quote prices simultaneously, and any remaining consumers make purchase decisions simultaneously again based on the updated beliefs about each other's type.

Our analysis focuses on equilibrium in which the buyers' period 1 behavior facing any price profile is sorted by their types. Specifically, under any price profile, we assume that the type space is divided into three intervals: the buyer types in the lowest interval who have the most favorable opinion about good $A$ choose $A$ in period 1 , those in the highest interval who have the most favorable opinion about good $B$ choose $B$ in period 1 , and those in the middle interval who have a moderate opinion about both goods defer their decisions until period $2 .^{3}$

The first key observation we make is on the intertemporal property of the prices on and off the equilibrium path. Specifically, we demonstrate that in equilibrium, the price path must be a martingale: for each firm, the ex ante expected value of its period 2 price equals its period 1 price both on and off the equilibrium path. This critical result is then extensively used in the derivation and characterization of an equilibrium. We first examine if the equilibrium can have the preemptive property. In other words, we examine if in equilibrium, any firm that successfully attracts one buyer in period 1 also sells to any remaining buyer in period 2. Although such preemption appears plausible given the strong position of the firm which wins a buyer in period 1, we show that it is not consistent with an equilibrium: Preemption requires the period 1 price to be very low, inducing either firm to profitably increase its period 1 price although it implies giving up the period 1 market share completely and concentrating on the period 2 market.

\footnotetext{
${ }^{2}$ We assume that the probability distribution of the type is independent between the consumers. This differentiates the present model from the standard models of social learning that suppose that the types are the noisy signals of the underlying state of the world.

${ }^{3}$ The thresholds between the intervals depend on the period 1 prices, and one or more of the intervals may be empty.
} 
This finding leads to the consideration of a strategy profile in which the losing firm (if any) in period 1 makes a sale in period 2 with positive probability. The main theorem of the paper constructs an equilibrium with this property taking advantage of the martingale property. We observe that the equilibrium period 1 price, which is uniquely determined, entails a discount compared with that in the one-period model to reflect the increased bargaining power of the buyers in the two-period model where they have a delay option. This discount is shown to be increasing in the degree of interdependence of the preferences. We can interpret this as the firms' response to the stronger incentive of the more interdependent consumers to delay their decisions. Interestingly, however, we also obtain a counter-intuitive conclusion that the probability of delay in equilibrium is decreasing in the degree of interdependence. As a natural consequence of this fact, we also find that the efficiency of the buyers' decisions in this equilibrium decreases with the degree of their interdependence.

The paper is organized as follows. After the discussion of the related literature in the next section, we formulate our model in Section 3. In Section 4, we analyze the equilibrium in the second period based on the sorting assumption of the buyer behavior in period 1. Section 5 derives the key martingale property of the price dynamics. We demonstrate the impossibility of the preemptive equilibrium in Section 6. Section 7 presents the main theorem of the paper that constructs the equilibrium. Characterizations of this equilibrium as well as comparative statics analysis are given in Section 8. We conclude with a discussion in Section 9.

\section{Related Literature}

Our model extends the standard models of dynamic durable good markets in at least two directions: First, we introduce interdependence in preferences between consumers which we consider essential for many durable goods as discussed above. Second, we introduce competition between the firms as a realistic feature of many durable goods markets.

The assumption on the interdependence of preferences in our model implies the presence of social learning by the consumers. In the social learning literature that begins with Banerjee (1992) and Bikhchandani et al. (1992), delay induced by learning is one of the central topics. Among others, Chamley and Gale (1994) and Gul and Lundholm (1995) present a model of strategic delay in the context of dynamic investment decisions. ${ }^{4}$ More recently, the literature on social learning looks at the sequential sales of a product

\footnotetext{
${ }^{4}$ See also Sgroi (2002) and Gunay (2008a, b). A textbook treatment of social learning and delay can be found in Chamley (2004).
} 
of uncertain quality by a monopolist, who optimally controls its price contingent on sales history. ${ }^{5}$ The standard assumption there is that each consumer makes a single decision: They either take a price offer, or else exit the market. Our model is new in that it combines the multiple purchase decisions and the strategic pricing of a product. Natural as it may appear, this combination has not been explored before to the best of our knowledge perhaps because of the substantial complications expected in the technical analysis. In particular, there is fundamental difficulty in checking the firms' deviation incentives in period 1 when those deviations change the buyers' delay incentives and also the outlook of the period 2 market. We show that the problem is solvable with the use of the martingale property mentioned in the Introduction.

The ability of consumers to wait and look for a better opportunity in later periods as examined here is the main theme of the literature on durable good monopoly that begins with the Coase conjecture. The subject is also extensively studied in the marketing literature on strategic consumers. ${ }^{6}$ The possibility that the buyers face uncertainty in their valuations is considered, among others, by Yu et al. (2011), and Bhalla (2012). ${ }^{7}$ Yu et al. (2011) study a two-period model of monopolistic sales when consumers learn about their valuations in the second period and the monopolist can control the number of products sold in each period. Bhalla (2012) studies a two-period model of monopolistic sales in which two consumers each observe a noisy signal about the binary product quality. When only consumer 1 is active in period 1 and may delay his decision until period 2, Bhalla (2012) shows that equilibrium pricing depends on the prior probability of the high quality product.

Problems in which firms with differentiated products compete in price for consumers who may delay their decisions are studied by Chen and Zhang (2009), Levin et al. (2009), and Liu and Zhang (2013). In Chen and Zhang (2009), the market consists of two segments that are loyal to either firm, and one segment that is opportunistic. Levin et al. (2009) also suppose that the market consists of multiple segments and that the valuation of each product is randomly determined every period. Liu and Zhang (2013) formulate a model of vertical product differentiation when consumer valuations are random but fixed over the periods. ${ }^{8}$

\footnotetext{
${ }^{5}$ See, for example, Bose et al. (2006, 2008), Aoyagi (2010), and Bhalla (2013).

${ }^{6}$ Beginning with Besanko and Winston (1990), one central question in this literature is what happens to the seller's revenue when the consumers become non-myopic and is given a chance to delay their decisions. See Gönsch et al. (2012) for a complete survey of the literature.

${ }^{7}$ Gunay (2013) considers a model in which the seller but not the buyers is privately informed of the quality of its good.

${ }^{8} \mathrm{Mak}$ et al. (2012) consider price competition when one buyer alternates between two sellers who
} 
To the best of our knowledge, however, the literature has only looked at the private value environment where each buyer's valuation is a function only of his own type or signal. Our model hence departs from the literature with the introduction of social learning based on interdependent valuations.

\section{Model}

Two risk neutral firms $A$ and $B$ sell durable goods $A$ and $B$, respectively, over two periods $t=1,2$ to two buyers $i=1,2$. Each buyer $i$ has private type $s_{i}$ that affects his and the other buyer's valuations of the two goods. Suppose that $s_{i}$ has a uniform distribution over the unit interval [0,1]. The value of a single unit of good $A$ to buyer $i$ is given by

$$
v_{i}=u+(1-k)\left(1-s_{i}\right)+k\left(1-s_{j}\right)=u+1-(1-k) s_{i}-k s_{j},
$$

and that of good $B$ is given by

$$
w_{i}=u+(1-k) s_{i}+k s_{j}
$$

where $u$ and $k$ are constants satisfying $0 \leq k<\frac{1}{2}$ and $u>\frac{1}{2}-k{ }^{9}$ When $k>0$, the two buyers' valuations of the goods are interdependent, and the larger is $k$, the more dependent buyers are on the other buyer's type. Since $k<1 / 2$, each buyer places more weight on his own type than the other buyer's type. ${ }^{10}$ On the other hand, when $k=0$, the valuations are independent. Note also that the value of good $B$ increases with both $s_{i}$ and $s_{j}$, whereas that of good $A$ decreases with them. Each buyer demands at most one unit of either good.

The game proceeds as follows: In period 1 , the two firms publicly and simultaneously quote prices $p_{A}^{1}$ and $p_{B}^{1}$ of their own goods. The two buyers then make simultaneous decisions on whether to buy either good or not buy and wait. If a buyer chooses to buy either good, then the decision is irreversible and he makes no further decision. The buyers' decisions in period 1 are publicly observed. If there is at least one buyer who chooses to wait in period 1 , the two firms again publicly and simultaneously quote prices $p_{A}^{2}$ and $p_{B}^{2}$ in period 2. Any buyer still in the market in period 2 then chooses to buy either good or not buy.

supply identical products.

${ }^{9}$ The latter condition ensures that the buyers' participation constraint does not bind in the period 2 price equilibrium. The multiplier $(1-k)$ is added to keep constant the range of valuations regardless of the value of $k$.

${ }^{10}$ The specification of valuations follows that in Aoyagi (2010). 
Each firm $f$ chooses its price $p_{f}^{t}$ in period $t$ from the set $\boldsymbol{R}_{+}$of non-negative real numbers, whereas each buyer $i$ makes his choice $d_{i}^{t}$ in period $t$ from the set $D=\{A, B, \emptyset\}$, where $d_{i}^{t}=\emptyset$ represents $i$ 's decision to make no purchase in period $t$. Any buyer $i$ who chooses to buy neither good in period 1 makes another decision in period 2 so that $d_{i}^{1}=\emptyset$ can alternatively be interpreted as the decision to wait. A period 1 history $h=\left(p^{1}, d^{1}\right)$ then consists of a pair $p^{1}=\left(p_{A}^{1}, p_{B}^{1}\right) \in \boldsymbol{R}_{+}^{2}$ of the prices quoted by the two firms as well as a pair $d^{1}=\left(d_{1}^{1}, d_{2}^{1}\right)$ of the decisions of the two buyers. Denote by $H=\boldsymbol{R}_{+}^{2} \times D^{2}$ the set of all period 1 histories. For $i=1,2$, let

$$
H_{i}=\left\{h=\left(p^{1}, d^{1}\right) \in H: d_{i}^{1}=\emptyset\right\}
$$

be the set of period 1 histories along which buyer $i$ waits, and

$$
H_{12}=H_{1} \cup H_{2}
$$

be the set of histories along which at least one buyer waits. Firm $f$ 's strategy consists of its price $\sigma_{f}^{1}$ in period 1 as well as the mapping $\sigma_{f}^{2}: H \rightarrow \boldsymbol{R}_{+}$that determines its period 2 price $p_{f}^{2}=\sigma_{f}^{2}(h)$ as a function of the period 1 history $h \in H$. On the other hand, buyer $i$ 's strategy is a mapping $\tau_{i}^{1}: S_{i} \times \boldsymbol{R}_{+}^{2} \rightarrow D$ that determines his period 1 choice as a function of his type $s_{i}$ and the period 1 prices $p^{1}$, along with a mapping $\tau_{i}^{2}: S_{i} \times \boldsymbol{R}_{+}^{2} \times H \rightarrow D$ that determines his period 2 choice as a function of his type $s_{i}$, the period 1 history $h$ as well as the period 2 price pair $p^{2}$. Since buyer $i$ has a decision to make in period 2 only if he chooses to wait in period 1 , we impose the restriction that $\tau_{i}^{2}\left(s_{i}, p^{2}, h\right)=\emptyset$ if $h \notin H_{i}$.

We will consider an equilibrium of this game which is a natural extension of perfect Bayesian equilibrium (PBE). Specifically, we impose an additional requirement that beliefs be obtained through Bayes rule from the buyers' strategies even when the period 1 price pair is off the path of play. ${ }^{11}$ The remainder of this section introduces some notation for the payoffs to present a formal description of the equilibrium. The uninterested reader can skip to the next section.

For any pair $p=\left(p_{A}, p_{B}\right)$ of prices and pair $s=\left(s_{1}, s_{2}\right)$ of types, let $\pi_{i}\left(s, p, d_{i}\right)$ denote buyer $i$ 's ex post payoff from decision $d_{i} \in D$ :

$$
\pi_{i}\left(s, p, d_{i}\right)= \begin{cases}v_{i}-p_{A} & \text { if } d_{i}=A \\ w_{i}-p_{B} & \text { if } d_{i}=B \\ 0 & \text { if } d_{i}=\emptyset .\end{cases}
$$

\footnotetext{
${ }^{11}$ Note that in the standard PBE, the belief is obtained through Bayes rule only along the equilibrium path. Our requirement would be implied by consistency in the definition of a sequential equilibrium which is defined for finite games.
} 
When the strategies of the firms and the buyers in period 2 are given, buyer $i$ 's ex post payoff over two periods as a function of his type as well as history $h=\left(p^{1}, d^{1}\right)$ is then written as:

$$
\Pi_{i}\left(s, p^{1}, d^{1} \mid \sigma^{2}, \tau^{2}\right)= \begin{cases}\pi_{i}\left(s, p^{1}, d_{i}^{1}\right) & \text { if } d_{i}^{1}=A \text { or } B, \\ \pi_{i}\left(s, \sigma^{2}(h), \tau_{i}^{2}\left(s_{i}, \sigma^{2}(h), h\right)\right) & \text { if } d_{i}^{1}=\emptyset,\end{cases}
$$

where $h=\left(p^{1}, d^{1}\right)$. Now for any history $h \in H$, let

$$
P_{i}(\cdot \mid h)
$$

denote the conditional distribution of buyer $i$ 's type $s_{i}$ given $h$. Each firm $f$ 's period 2 payoff from buyer $i$ is expressed as a function of the period 2 price pair $p^{2}$ as well as when history $h$ and buyer $i$ 's period 2 strategies $\tau_{i}^{2}$ :

$$
\pi_{f, i}^{2}\left(p^{2} \mid \tau_{i}^{2}, h\right)=p_{f}^{2} P_{i}\left(\tau_{i}^{2}\left(s_{i}, p^{2}, h\right)=f \mid h\right)
$$

Furthermore, when the two buyers' strategies $\tau=\left(\tau^{1}, \tau^{2}\right)$ in both periods as well as the firms' strategies $\sigma^{2}=\left(\sigma_{A}^{2}, \sigma_{B}^{2}\right)$ in period 2 are given, let $\Pi_{f, i}\left(p^{1} \mid \tau, \sigma^{2}\right)$ denote firm $f$ 's payoff over two periods from buyer $i$ as a function of the period 1 price pair:

$$
\Pi_{f, i}\left(p^{1} \mid \tau_{i}, \sigma^{2}\right)=p_{f}^{1} P\left(\tau_{i}^{1}\left(s_{i}, p^{1}\right)=f\right)+E\left[\pi_{f, i}^{2}\left(\sigma^{2}(h) \mid \tau^{2}, h\right)\right],
$$

where $h=\left(p^{1}, \tau_{1}^{1}\left(s_{1}, p^{1}\right), \tau_{2}^{1}\left(s_{2}, p^{1}\right)\right)$. Firm $f^{\prime}$ 's per buyer payoffs from both buyers in period 2 and over two periods are then given by

$$
\pi_{f}^{2}\left(p^{2} \mid \tau^{2}, h\right)=\frac{1}{2} \sum_{i=1}^{2} \pi_{f, i}^{2}\left(p^{2} \mid \tau_{i}^{2}, h\right), \quad \text { and } \quad \Pi_{f}\left(p^{1} \mid \tau, \sigma^{2}\right)=\frac{1}{2} \sum_{i=1}^{2} \Pi_{f, i}\left(p^{1} \mid \tau, \sigma^{2}\right),
$$

respectively.

In period 2, for any type $s_{i}$, history $h \in H_{i}$, and period 2 price pair $p^{2}$, buyer $i$ 's decision $\tau_{i}^{2}\left(s_{i}, p^{2}, h\right) \in D$ in period 2 maximizes his expected utility, and for any $h \in H_{12}$ along which at least one buyer chooses to wait in period 1, the firms' price pair $\sigma^{2}(h)$ in period 2 is a NE of the firms' game in period 2 given the belief $P_{i}(\cdot \mid h)$ about each buyer $i$ 's type conditional on $h$. Formally, for each $i=1,2, h \in H_{i}$ and $p^{2} \in \boldsymbol{R}_{+}^{2}, \tau_{i}^{2}$ is sequentially rational and satisfies

$$
\tau_{i}^{2}\left(s_{i}, p^{2}, h\right) \in \underset{d_{i}^{2}}{\operatorname{argmax}} E_{s_{j}}\left[\pi_{i}\left(s, p^{2}, d_{i}^{2}\right) \mid s_{i}, h\right],
$$

and for each $h \in H_{12}$ and $\tau^{2}$ that is sequentially rational, $\sigma^{2}(h)$ is sequentially rational and satisfies for $f=A, B$, and $\ell \neq f$,

$$
\sigma_{f}^{2}(h) \in \underset{p_{f}^{2}}{\operatorname{argmax}} \pi_{f}^{2}\left(p_{f}^{2}, \sigma_{\ell}^{2}(h) \mid \tau^{2}, h\right) .
$$


Furthermore, facing any price pair $p^{1}$, buyer $i$ 's period 1 strategy $\tau_{i}^{1}$ is sequentially rational given the sequentially rational period 2 strategies $\tau^{2}$ and $\sigma^{2}$ : For every type $s_{i}$,

$$
\tau_{i}^{1}\left(s_{i}, p^{1}\right) \in \underset{d_{i}^{1}}{\operatorname{argmax}} E\left[\Pi_{i}\left(s_{i}, p^{1}, d_{i}^{1} \mid \sigma^{2}, \tau^{2}\right)\right],
$$

and the price pair $\sigma^{1}$ is a NE of the firms' game given the buyers' strategies and the firms' period 2 strategies both of which are sequentially rational: For $f=A, B$, and $\ell \neq f$,

$$
\sigma_{f}^{1} \in \underset{p_{f}^{1}}{\operatorname{argmax}} \Pi_{f}\left(p_{f}^{1}, \sigma_{\ell}^{1} \mid \tau, \sigma^{2}\right) .
$$

Finally, the conditional distribution $P_{i}(\cdot \mid h)$ about buyer $i$ 's type $s_{i}$ given history $h=$ $\left(p^{1}, d^{1}\right) \in H$ is derived through Bayes rule whenever a strictly positive measure of types of buyer $i$ choose $d_{i}^{1}$ when faced with $p^{1}: P_{i}\left(\tau_{i}^{1}\left(s_{i}, p^{1}\right)=d_{i}^{1}\right)>0 . P_{i}(\cdot \mid h)$ is arbitrary otherwise.

\section{Equilibrium in Period 2}

We begin by solving for an equilibrium in period 2. Consider buyer $i$ 's problem in period 2 following history $h \in H_{i}$ along which he chooses to wait $d_{i}^{1}=\emptyset$ in period 1 . Let $e_{j}(h)$ be the expected value of buyer $j$ 's type $s_{j}$ implied by the period 1 history $h$ :

$$
e_{j}(h)=E_{s_{j}}\left[s_{j} \mid h\right]=E_{s_{j}}\left[s_{j} \mid p^{1}, d_{j}^{1}\right],
$$

where the conditional expectation $E_{s_{j}}[\cdot \mid h]$ given $h$ is taken with respect to the conditional distribution $P_{s_{j}}(\cdot \mid h)$ given $h$. Facing the price pair $p^{2}$ in period 2, buyer $i$ chooses $A$ in period 2 if

$$
u+1-(1-k) s_{i}-k e_{j}(h)-p_{A}^{2}>\max \left\{u+(1-k) s_{i}+k e_{j}(h)-p_{B}^{2}, 0\right\},
$$

chooses $B$ if

$$
u+(1-k) s_{i}+k e_{j}(h)-p_{B}^{2}>\max \left\{u+1-(1-k) s_{i}-k e_{j}(h)-p_{A}^{2}, 0\right\},
$$

and chooses $\emptyset$ if

$$
0>\max \left\{u+1-(1-k) s_{i}-k e_{j}(h)-p_{A}^{2}, u+(1-k) s_{i}+k e_{j}(h)-p_{B}^{2}\right\} .
$$

In any PBE, hence, buyer $i$ 's period 2 strategy $\tau_{i}^{2}$ along any history $h \in H_{i}$ must satisfy

$$
\tau_{i}^{2}\left(s_{i}, p^{2}, h\right)= \begin{cases}A & \text { if } s_{i}<\min \left\{\frac{1-2 k e_{j}(h)-p_{A}^{2}+p_{B}^{2}}{2(1-k)}, \frac{u+1-k e_{j}(h)-p_{A}^{2}}{1-k}\right\}, \\ B & \text { if } s_{i}>\max \left\{\frac{1-2 k e_{j}(h)-p_{A}^{2}+p_{B}^{2}}{2(1-k)}, \frac{-u-k e_{j}(h)+p_{B}^{2}}{1-k}\right\}, \\ \emptyset & \text { if } \frac{u+1-k e_{j}(h)-p_{A}^{2}}{1-k}<s_{i}<\frac{-u-k e_{j}(h)+p_{B}^{2}}{1-k} .\end{cases}
$$


Consider next the firms' game in period 2 following $h \in H_{i}$ along which buyer $i$ chooses to wait in period 1. It follows from (2) that firm $A$ 's period 2 payoff from buyer $i$ along $h \in H_{i}$ is given by

$$
\pi_{A, i}^{2}\left(p^{2} \mid \tau_{i}^{2}, h\right)=p_{A}^{2} P_{i}\left(s_{i}<\min \left\{\frac{1-2 k e_{j}(h)-p_{A}^{2}+p_{B}^{2}}{2(1-k)}, \frac{u+1-k e_{j}(h)-p_{A}^{2}}{1-k}\right\} \mid h\right) .
$$

Likewise, firm $B$ 's period 2 payoff from buyer $i$ is given by

$$
\pi_{B, i}^{2}\left(p^{2} \mid \tau^{2}, h\right)=p_{B}^{2} P_{i}\left(s_{i}>\max \left\{\frac{1-2 k e_{j}(h)-p_{A}^{2}+p_{B}^{2}}{2(1-k)}, \frac{-u-k e_{j}(h)+p_{B}^{2}}{1-k}\right\} \mid h\right) .
$$

We assume throughout that the buyers' period 1 strategies are such that for any price pair $p^{1}$, there exist $x\left(p^{1}\right)$ and $y\left(p^{1}\right)$ with $0 \leq x\left(p^{1}\right) \leq y\left(p^{1}\right) \leq 1$ such that

$$
\tau_{i}\left(s_{i}, p^{1}\right)= \begin{cases}A & \text { if } s_{i}<x\left(p^{1}\right), \\ \emptyset & \text { if } x\left(p^{1}\right)<s_{i}<y\left(p^{1}\right), \\ B & \text { if } s_{i}>y\left(p^{1}\right) .\end{cases}
$$

In other words, when faced with $p^{1}$, buyer $i$ chooses $A$ if his type is at the lower-end of the type space, $B$ if it is at the higher-end, and $\emptyset$ if it is in the middle. For simplicity, we often omit the dependence of the thresholds on $p^{1}$ and simply write them as $x$ and $y$.

Although we assume that the buyers do not discount the period 2 payoffs, our justification of this sorting assumption comes from the observation that when they do, their period 1 strategy must take this form in any equilibrium. Specifically, let $\delta \in(0,1]$ denote their discount factor and suppose that when they buy either good in period 2 , the value of the good as well as its price is discounted by $\delta$. For example, when buyer $i$ buys $A$ in period 1 for price $p_{A}^{1}$, his payoff equals $v_{i}-p_{A}^{1}$, but when he buys it in period 2 for price $p_{A}^{2}$, his payoff equals $\delta\left(v_{i}-p_{A}^{2}\right)$. We have the following observation in this case.

Lemma 1. Suppose that $(\sigma, \tau, P)$ is an equilibrium under positive discounting $\delta<1$ by the buyers. For any buyer $i$ and period 1 price profile $p^{1}, \tau_{i}^{1}$ satisfies (3) for some $x=x\left(p^{1}\right)$ and $y=y\left(p^{1}\right)$ such that $0 \leq x \leq y \leq 1$.

The intuition behind Lemma 1 is as follows: Suppose there is some type $s_{i}$ for whom choosing $A$ in period 1 is optimal. Consider any type $s_{i}^{\prime}<s_{i}$. First, no such $s_{i}^{\prime}$ will choose $B$ in period 1 . Second, if $s_{i}^{\prime}$ waits and chooses $A$ in period 2 after some move by $j$ in period 1 , then his payoff conditional on that event is strictly lower than choosing $A$ in period 1 because of positive discounting. Third, if he waits and chooses $B$ after 
some move by $j$, then his payoff conditional on that event is strictly lower than the corresponding payoff of type $s_{i}$. It follows that type $s_{i}^{\prime}$ strictly prefers choosing $A$ to waiting in period 1 .

(3) need not hold under no discounting since choosing $A$ in period 1 may yield the same payoff as waiting and then unconditionally choosing $A$ in period 2 not just for a single type but for a range of types. However, Lemma 1 shows that if we require the buyer behavior to be continuous at $\delta=1$, then it should satisfy (3).

When buyer $j$ 's decision is described by $(3)$, then $e_{j}(h)$ defined in (1) equals:

$$
e_{j}(h)= \begin{cases}\frac{x}{2} & \text { if } d_{j}^{1}=A, \\ \frac{x+y}{2} & \text { if } d_{j}^{1}=\emptyset \\ \frac{1+y}{2} & \text { if } d_{j}^{1}=B .\end{cases}
$$

Suppose that both buyers use the same period 1 strategy (3) with $x<y$. Then the conditional probability $P_{i}(\cdot \mid h)$ given $h \in H_{i}$ is the uniform distribution over the interval $(x, y)$. Hence, firm $f$ 's expected payoff $\pi_{f, i}^{2}$ from buyer $i$ in period 2 can be explicitly given as in the proof of Lemma 2 in the Appendix. Furthermore, given the symmetry between the buyers' strategies, firm $f$ 's (per buyer) payoff $\pi_{f}^{2}$ from both buyers equals $\pi_{f, i}^{2}$ after any history $h \in H_{1} \cap H_{2}$ along which they both wait in period 1 . Based on these payoff functions, the following lemma identifies the equilibrium of the firms' game in period 2.

Lemma 2. Suppose that for some $p^{1}$, the buyers' behavior in period 1 is described by (3) for $x=x\left(p^{1}\right)<y\left(p^{1}\right)=y$. Then the equilibrium price profile $\left(\sigma_{A}^{2}(h), \sigma_{B}^{2}(h)\right)$ in period 2 following history $h=\left(p^{1}, d^{1}\right) \in H_{12}$ is unique and given as follows:

a) (interior equilibrium) If $1-2 k e_{j}(h) \in[2(1-k)(2 x-y), 2(1-k)(2 y-x)],{ }^{12}$ then

$$
\begin{aligned}
& \left(\sigma_{A}^{2}(h), \sigma_{B}^{2}(h)\right) \\
& =\left(\frac{1-2 k e_{j}(h)+2(1-k)(y-2 x)}{3}, \frac{-1+2 k e_{j}(h)+2(1-k)(2 y-x)}{3}\right),
\end{aligned}
$$

and the two firms segment the market with firm $A$ capturing $\left(x, \frac{1-2 k e_{j}(h)}{6(1-k)}+\frac{x+y}{3}\right)$ and firm $B$ capturing $\left(\frac{1-2 k e_{j}(h)}{6(1-k)}+\frac{x+y}{3}, y\right)$.

b) (A-corner equilibrium) If $1-2 k e_{j}(h)>2(1-k)(2 y-x)$, then

$$
\left(\sigma_{A}^{2}(h), \sigma_{B}^{2}(h)\right)=\left(1-2 k e_{j}(h)-2(1-k) y, 0\right),
$$

and firm A monopolizes the market by capturing $(x, y)$.

\footnotetext{
${ }^{12}$ Since $y \geq x, 2(1-k)(2 x-y) \leq 2(1-k)(2 y-x)$.
} 
c) (B-corner equilibrium) If $1-2 k e_{j}(h)<2(1-k)(2 x-y)$, then

$$
\left(\sigma_{A}^{2}(h), \sigma_{B}^{2}(h)\right)=\left(0,-1+2 k e_{j}(h)+2(1-k) x\right),
$$

and firm B monopolizes the market by capturing $(x, y)$.

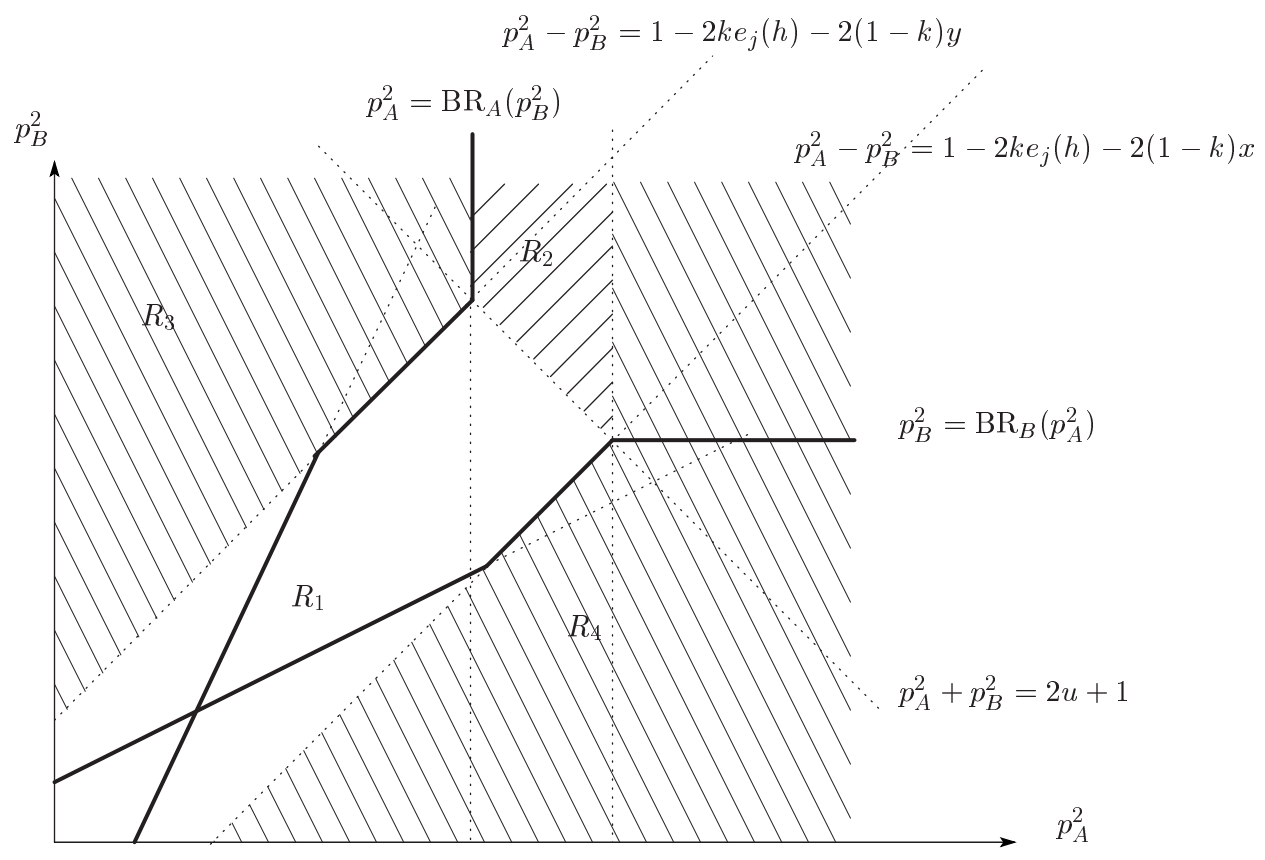

Figure 1: Best-response diagram in period 2: interior equilibrium

Figure 1 illustrates the best-response correspondences and the equilibrium price profile when it is in the interior (Case (a)). Lemma 2 also characterizes the equilibrium of the one-period game in which the firms quote prices once and the buyers make a single purchase decision since such a game is equivalent to the period 2 game with $x=0, y=1$ and $e_{j}(h)=1 / 2$.

Proposition 3. In the one-period game, the equilibrium price profile is unique and given by $\left(p_{A}, p_{B}\right)=(1-k, 1-k)$, and the firms segment the market with firm A capturing $[0,1 / 2)$ and firm $B$ capturing $(1 / 2,1]$.

Proposition 3 shows that the higher the dependence parameter $k$, the more intense is the competition between the firms and the lower is the equilibrium price. 


\section{$5 \quad$ Equilibrium Price Dynamics}

In this section, we make some critical observation on the relationship between the period 1 price and the period 2 prices. Specifically, suppose that the period 1 price is $p^{1}$ and that the buyers' period 1 strategies are given by (3). While the period 2 prices take different values depending on the buyers' decisions in period 1 , we show that in equilibrium, for any price quote $p_{f}^{1}$ by firm $f$ in period 1 on or off the equilibrium path, the expected value of its period 2 price must equal $p_{f}^{1}$ after the history $h=\left(p^{1}, d^{1}\right) \in H_{12}$. The derivation of the equilibrium of the full game in the next section fully exploits this martingale property of the price dynamics.

Lemma 4. (Martingale property) Suppose that $(\sigma, \tau, P)$ is an equilibrium, and that the buyers' period 1 strategies $\tau^{1}$ satisfies (3) for any period 1 price pair $p^{1}=\left(p_{A}^{1}, p_{B}^{1}\right)$. Then for any $p^{1}$, if $x=x\left(p^{1}\right) \in(0,1)$, then the expected price of $A$ buyer $i$ will face in period 2 when he waits equals $p_{A}^{1}$. Likewise, if $y=y\left(p^{1}\right) \in(0,1)$, then the expected price of $B$ buyer $i$ faces in period 2 when he waits equals $p_{B}^{1}$ :

$$
\begin{aligned}
& p_{A}^{1}=E\left[\sigma_{B}^{2}\left(p^{1}, \emptyset, d_{j}^{1}\right)\right], \\
& p_{B}^{1}=E\left[\sigma_{B}^{2}\left(p^{1}, \emptyset, d_{j}^{1}\right)\right] .
\end{aligned}
$$

Lemma 4 is not an artifact of our assumption that the type distribution is uniform. Rather, it follows from the general observation that the critical type $x$ at the lower end of the interval in period 2 is indifferent between the two goods in the $B$-corner equilibrium, and that the type $y$ at the higher end is indifferent between the two goods in the $A$-corner equilibrium. ${ }^{13}$ More specifically, the intuition behind Lemma 4 is as follows: Depending on the decision of buyer $j$ in period 1 , buyer $i$ of the critical type $x$ may face either the interior equilibrium or one of the corner equilibria in period 2 if he chooses to wait. If he chooses $B$ in period 2 after some decision by $j$, it implies that the $B$-corner equilibrium is in play. Then his payoff from choosing $A$ is just the same as that from choosing $B$ given the property of such an equilibrium. That is, for type $x$, making an optimal contingent choice in period 2 is equivalent to making an unconditional choice of $A$ then. On the other hand, if he always chooses $A$ in period 2 regardless of $j$ 's period 1 decision, then by the law of iterated expectation, the his ex ante expected valuation of good $A$ in period 2 is just the same as that in period 1. Given that type $x$ is indifferent between choosing

\footnotetext{
${ }^{13}$ Lemma 4 is reminiscent of the martingale property found in the sequential auction model of Weber (1981) although the logic here is unrelated.
} 
$A$ today and waiting, the expected price in period 2 must then be the same as the price in period 1. The symmetric argument for type $y$ yields the property of the price of $B$.

Equilibrium price dynamics is one central topic in the literature on dynamic sales. Bose et al. (2008) and Bhalla (2013) both show in their respective sequential sales models that the price path is a super-martingale in the sense that the ex ante expected prices go down with the progress of sales. In a two-period model in which only one consumer arrives in period 1 and may delay, on the other hand, Bhalla (2012) shows that the prices can either increase or decrease over periods depending on the prior belief about the quality of the good. In a model of online sales with random arrival of consumers, Gallien (2006) shows that the price path is a sub-martingale. While the price dynamics in these models is a derivative property of an equilibrium, the martingale property in our model is the key building block of an equilibrium as seen below and must hold both on and off the equilibrium path.

\section{Impossibility of a Preemptive Equilibrium}

Before turning to the analysis of the full model, it is useful to first examine the buyer behavior in the simplified model in which the period 2 prices are not contingent on the outcome of period 1. Specifically, suppose that the symmetric price profile such that $p_{A}^{1}=p_{B}^{1}$ in period 1 and $p_{A}^{2}=p_{B}^{2}$ in period 2 is exogenously given. If we write $x=x\left(p^{1}\right)$ and $y=y\left(p^{1}\right)$ in (3), then by symmetry, $y=1-x$. Clearly, full delay (i.e., $x=0$ ) takes place if $p_{A}^{1}>p_{A}^{2}$. Hence, suppose that $\Delta=p_{A}^{2}-p_{A}^{1} \geq 0$. If $\Delta>0$, a buyer may delay his decision only if he intends to make different choices depending on the outcome of period 1. Specifically, when $\Delta>0$, it must be the case that when buyer $i$ waits but buyer $j$ chooses either product in period $1, i$ 's period 2 choice mimics $j$ 's choice in period 1 . In this case, any type $s_{i} \in(x, 1-x)$ prefers waiting to choosing $A$ in period 1 if

$$
\begin{aligned}
1-(1-k) s_{i}-k \frac{1}{2}-p_{A}^{1} & \leq x\left\{1-(1-k) s_{i}-k \frac{x}{2}-p_{A}^{2}\right\} \\
& +(1-2 x)\left\{1-(1-k) s_{i}-k \frac{1}{2}-p_{A}^{2}\right\} \\
& +x\left\{(1-k) s_{i}+k\left(1-\frac{x}{2}\right)-p_{B}^{2}\right\} .
\end{aligned}
$$

The left-hand side is $i$ 's payoff from choosing $A$ today while the right-hand side is his payoff from waiting: The three terms correspond to $i$ 's choice of $A, A$, and $B$ in period 2 when $j$ 's decision in period 1 is $A, \emptyset$, and $B$, respectively. Since type $s_{i}=x$ is indifferent between $A$ and waiting, the equality should hold in (8) for $s_{i}=x$. Upon simplification, 
this equality is equivalent to

$$
(2-3 k) x^{2}-(1-2 k) x-\Delta=0 .
$$

Solving for $x$ satisfying $x \in\left(0, \frac{1}{2}\right)$, we obtain

$$
x= \begin{cases}\frac{1-2 k+\sqrt{(1-2 k)^{2}+4(2-3 k) \Delta}}{2(2-3 k)} & \text { if } \Delta \in\left(0, \frac{k}{4}\right], \\ \frac{1-2 k}{2-3 k} & \text { if } \Delta=0 .\end{cases}
$$

While the buyer behavior is uniquely described by (9) when $\Delta>0$, when $\Delta=0$, we can also verify that any $x \in\left[0, \frac{1-2 k}{2-3 k}\right]$ is also a valid threshold. ${ }^{14}$ In this case, every type $s_{i} \in\left(x, \frac{1-2 k}{2-3 k}\right)$ chooses $A$ in period 2 regardless of $j$ 's decision in period 1 , and hence is indifferent between waiting and choosing $A$ in period 1 . Note, however, that only $x=\frac{1-2 k}{2-3 k}$ describes behavior that is continuous in $\Delta$ at $\Delta=0$. The following proposition summarizes these observations.

Proposition 5. (Buyer behavior along a fixed price path) Suppose that the buyer behavior is described by (3) under the symmetric and fixed price profile $\left(p^{1}, p^{2}\right)$. Then $x$ is uniquely given by (9) when $\Delta=p_{A}^{2}-p_{A}^{1}>0$, and $x \in\left[0, \frac{1-2 k}{3-2 k}\right]$ when $\Delta=0$. For $x$ given in (9), if buyer $i$ waits and buyer $j$ moves in period 1, then $i$ chooses the same firm as $j$ in period 2, and the probability of delay $1-2 x$ is increasing in $k$.

We now proceed to the analysis of the equilibrium of our original model in which the period 2 prices are contingent on the period 1 outcome. In this section, we examine whether or not the equilibrium can be preemptive in the sense that the firm which successfully attracts one buyer in period 1 also attracts any remaining buyer in period 2 . In other words, along the equilibrium path, the choice of $A$ by a single buyer in period 1 is followed by the $A$-corner equilibrium in period 2, and the choice of $B$ is followed by the $B$-corner equilibrium in period 2. As seen in Proposition 5, such a property characterizes buyer behavior under the fixed price path.

Suppose that $(\sigma, \tau, P)$ is a symmetric preemptive equilibrium. Denote the critical types in (3) by $x^{*}=x\left(\sigma^{1}\right)$ and $y^{*}=y\left(\sigma^{1}\right)$ under the equilibrium price profile $\sigma^{1}$ in period 1. By symmetry, we have $y^{*}=1-x^{*}$.

\footnotetext{
${ }^{14}$ This follows since for any such $x$, type $s_{i}<x$ finds $A$ better than $B$ even after $j$ 's choice of $B$ in period 1:$$
1-(1-k) s_{i}-k \frac{1+y}{2}-p_{A}^{2} \leq(1-k) s_{i}+k \frac{1+y}{2}-p_{B}^{2}
$$ 
First, by Lemma 2, the $A$-corner equilibrium is played after $h=\left(\sigma^{1}, d^{1}\right)$ with $d^{1}=$ $(\emptyset, A)$ if and only if

$$
1-2 k\left(\frac{x^{*}}{2}\right) \geq 2(1-k)\left\{2\left(1-x^{*}\right)-x^{*}\right\}
$$

This along with $y^{*}=1-x^{*} \geq x^{*}$ implies that the relevant range of $x^{*}$ is given by

$$
x^{*} \in\left[\frac{3-4 k}{6-7 k}, \frac{1}{2}\right] \text {. }
$$

By symmetry, this condition is also necessary and sufficient for the $B$-corner equilibrium to be played after $h=\left(\sigma^{1}, d^{1}\right)$ with $d^{1}=(\emptyset, B)$.

Next, by the martingale property (Lemma 4 ), the period 1 price $\sigma_{A}^{1}\left(=\sigma_{B}^{1}\right)$ equals the expected equilibrium price in period 2 :

$$
\begin{aligned}
\sigma_{A}^{1} & =x^{*}\left\{1-k x^{*}-2(1-k)\left(1-x^{*}\right)\right\} \\
& +\left(1-2 x^{*}\right) \frac{1-k+2(1-k)\left(1-3 x^{*}\right)}{3} \\
& +x^{*} \cdot 0 \\
& =(6-7 k)\left(x^{*}\right)^{2}-(5-6 k) x^{*}+1-k .
\end{aligned}
$$

When $x^{*}$ satisfies (10), the range of $\sigma_{A}^{1}$ is given by

$$
\sigma_{A}^{1} \in\left[\frac{k(1-k)}{6-7 k}, \frac{k}{4}\right]
$$

Hence, the period 1 price in a preemptive equilibrium, if any, must be significantly lower than, say, the equilibrium price $1-k$ in the one-period model. The following proposition shows that this low price gives either firm an incentive to deviate and increase its price. In fact, setting a sufficiently high price in period 1 is a profitable deviation although it implies giving up the market share in period 1.

Proposition 6. (Impossibility of a Preemptive Equilibrium) Suppose that the buyers behavior in period 1 is described by (3). Then there exists no symmetric equilibrium $(\sigma, \tau, P)$ such that the on-the-path period 1 history $h=\left(\sigma^{1}, d^{1}\right)$ induces the A-corner equilibrium if $d^{1}=(\emptyset, A)$ and the $B$-corner equilibrium if $d^{1}=(\emptyset, B)$.

\section{Existence of a Non-Preemptive Equilibrium}

Having seen in the previous section that the equilibrium cannot be preemptive, we turn to the alternative possibility where the period 2 equilibrium is always an interior 
equilibrium. In other words, even if only one firm wins a buyer in period 1, some buyer types still choose the other firm in period 2. In this section, we present the main theorem of the paper that proves the existence of such a non-preemptive equilibrium.

Recall that $H_{i}$ is the set of histories along which buyer $i$ waits in period 1. Suppose now that for some period 1 price pair $p^{1}$, every history $h=\left(p^{1}, d^{1}\right) \in H_{i}$ induces an interior equilibrium in period 2. By Lemma 2(a), this holds if and only if

$$
\begin{aligned}
2(1-k)(2 x-y) & \leq 1-2 k e_{j}\left(p^{1}, \emptyset, B\right) \\
& <1-2 k e_{j}\left(p^{1}, \emptyset, \emptyset\right) \\
& <1-2 k e_{j}\left(p^{1}, \emptyset, A\right) \leq 2(1-k)(2 y-x) .
\end{aligned}
$$

Substituting $e_{j}\left(p^{1}, \emptyset, A\right)=\frac{x}{2}$ and $e_{j}\left(p^{1}, \emptyset, A\right)=\frac{1+y}{2}$ and rearranging, we see that these conditions are equivalent to

$$
4(1-k) x-(2-3 k) y \leq 1-k \quad \text { and } \quad(2-3 k) x-4(1-k) y \leq-1 .
$$

Since $E\left[e_{j}(h)\right]=\frac{1}{2}$, the expected price that buyer $i$ will face in period 2 is then given by

$$
\begin{aligned}
E\left[\sigma_{A}^{2}\left(p^{1}, d_{i}^{1}=\emptyset, d_{j}^{1}\right)\right] & =\frac{1-2 k E\left[e_{j}(h)\right]+2(1-k)(y-2 x)}{3} \\
& =(1-k) \frac{1+2(y-2 x)}{3}, \\
E\left[\sigma_{B}^{2}\left(p^{1}, d_{i}^{1}=\emptyset, d_{j}^{1}\right)\right] & =\frac{-1+2 k E\left[e_{j}(h)\right]+2(1-k)(2 y-x)}{3} \\
& =(1-k) \frac{-1+2(2 y-x)}{3} .
\end{aligned}
$$

Hence, by the martingale property (Lemma 4),

$$
p_{A}^{1}=(1-k) \frac{1+2(y-2 x)}{3},
$$

and

$$
p_{B}^{1}=(1-k) \frac{-1+2(2 y-x)}{3} .
$$

Define

$$
q_{A}=\frac{p_{A}^{1}}{1-k} \quad \text { and } \quad q_{B}=\frac{p_{B}^{1}}{1-k}
$$

to be the period 1 prices adjusted by the degree of interdependence. We then have

$$
q_{A}=\frac{1}{3}(1-4 x+2 y), \quad \text { and } \quad q_{B}=\frac{1}{3}(-1+4 y-2 x) .
$$


Solving for $x$ and $y$ yields:

$$
\begin{aligned}
& x=\frac{1}{2}-\frac{2 q_{A}-q_{B}}{2}, \\
& y=\frac{1}{2}+\frac{2 q_{B}-q_{A}}{2} .
\end{aligned}
$$

Write $\pi_{f}^{2 *}(h)=\pi_{f}^{2}\left(\sigma^{2}(h) \mid \tau^{2}, h\right)$ for firm $f$ 's (per buyer) payoff in period 2 along the history $h=\left(p^{1}, d^{1}\right)$ when the equilibrium strategies $\sigma^{2}$ and $\tau^{2}$ are played in period 2. It readily follows from Lemma 2 that $\pi_{f}^{2 *}(h)$ for $h=\left(p^{1}, d^{1}\right) \in H_{12}$ is given by

$$
\begin{aligned}
& \pi_{A}^{2 *}(h)=\frac{1}{y-x} \frac{\left\{1-2 k e_{j}(h)+2(1-k)(y-2 x)\right\}^{2}}{18(1-k)}, \\
& \pi_{B}^{2 *}(h)=\frac{1}{y-x} \frac{\left\{1-2 k e_{j}(h)+2(1-k)(2 y-x)\right\}^{2}}{18(1-k)} .
\end{aligned}
$$

Firm $f$ 's (per buyer) payoff $\Pi_{f}$ over two periods is then written as:

$$
\begin{aligned}
& \Pi_{A}\left(p^{1} \mid \sigma^{2}, \tau\right)=p_{A}^{1} x+(y-x) {\left[x \pi_{A}^{2 *}\left(p^{1}, \emptyset, A\right)\right.} \\
&+\left.(y-x) \pi_{A}^{2 *}\left(p^{1}, \emptyset, \emptyset\right)+(1-y) \pi_{A}^{2 *}\left(p^{1}, \emptyset, B\right)\right], \\
& \Pi_{B}\left(p^{1} \mid \sigma^{2}, \tau\right)=p_{B}^{1}(1-y)+(y-x)\left[x \pi_{B}^{2 *}\left(p^{1}, \emptyset, A\right)\right. \\
&\left.+(y-x) \pi_{B}^{2 *}\left(p^{1}, \emptyset, \emptyset\right)+(1-y) \pi_{B}^{2 *}\left(p^{1}, \emptyset, B\right)\right] .
\end{aligned}
$$

Substitution of $\pi_{A}^{2 *}$ into (18) yields upon simplification

$$
\begin{aligned}
& \Pi_{A}\left(p^{1} \mid \sigma^{2}, \tau\right) \\
& =p_{A}^{1} x+\frac{1}{18(1-k)}\left[(1-k)^{2}\{1+2(y-2 x)\}^{2}+k^{2} y(1-x)(1+x-y)\right] .
\end{aligned}
$$

Now rewrite $\Pi_{f}$ as a function of $\left(q_{A}, q_{B}\right): \hat{\Pi}_{f}\left(q_{A}, q_{B}\right)=\Pi_{f}\left(p^{1} \mid \sigma_{2}, \tau\right)$. Substituting (17), we obtain

$$
\begin{aligned}
\hat{\Pi}_{A}\left(q_{A}, q_{B}\right) & =(1-k) q_{A}\left(\frac{1}{2}-\frac{2 q_{A}-q_{B}}{2}\right)+\frac{1-k}{2} q_{A}^{2} \\
& +\frac{k^{2}}{18(1-k)}\left(\frac{1}{2}+\frac{2 q_{B}-q_{A}}{2}\right)\left(\frac{1}{2}+\frac{2 q_{A}-q_{B}}{2}\right)\left(1-\frac{q_{A}+q_{B}}{2}\right)
\end{aligned}
$$

Suppose now that $\sigma^{1}=\left(\sigma_{A}^{1}, \sigma_{B}^{1}\right)$ is the equilibrium price pair in period 1. If for any price pair $p^{1}$ in the neighborhood of $\sigma^{1}$, every history $h=\left(p^{1}, d^{1}\right) \in H_{i}$ induces an interior equilibrium in period 2, then we can identify the equilibrium price pair $\sigma^{1}$ as a solution to the first-order conditions of the maximization of $\hat{\Pi}_{A}$ and the corresponding payoff function $\hat{\Pi}_{B}$ of firm $B$. Furthermore, if the equilibrium price pair is symmetric $\left(q_{A}, q_{B}\right)=(q, q)$, then $q$ must satisfy $\frac{\partial \hat{\Pi}_{A}}{\partial q_{A}}(q, q)=0$. The following theorem identifies an equilibrium through this consideration. 
Theorem 7. (Non-preemptive equilibrium) Let q be given by

$$
q= \begin{cases}\frac{-36(1-k)^{2}-k^{2}+\sqrt{\left\{36(1-k)^{2}+k^{2}\right\}^{2}+3 k^{2}\left\{72(1-k)^{2}+k^{2}\right\}}}{3 k^{2}} & \text { if } k>0, \\ 1 & \text { if } k=0 .\end{cases}
$$

There exists a symmetric equilibrium $(\sigma, \tau, P)$ in which the firms quote $\sigma_{A}^{1}=\sigma_{B}^{1}=$ $(1-k) q$ in period 1 and the buyers' period 1 strategies $\tau_{i}^{1}$ on the equilibrium path are described by (3) for $x=\frac{1-q}{2}$ and $y=\frac{1+q}{2}$.

The proof in the Appendix constructs the equilibrium by specifying the buyer response to every off-equilibrium price pair in period 1 . For a period 1 price pair that corresponds to a unilateral deviation, this construction determines the profitability of the deviation. For illustration, suppose that firm $A$ unilaterally deviates and slightly cuts its price in period 1 . This deviation is followed by higher values of the thresholds $x$ and $y$ : More buyer types immediately choose $A$, and less buyer types immediately choose $B$. These thresholds then determine the active buyer types in the period 2 market and the payoff of the deviating firm there. Hence, the profitability of the price cut in period 1 depends on the change in immediate sales in period 1 as well as on the change in the payoff in period 2, both of which are caused by the change in the thresholds $x$ and $y$. Evaluation of the profitability of a deviation hence requires the exact identification of the thresholds based on the martingale property.

Given that the equilibrium price in the one period model equals $1-k$ as seen earlier, we can interpret $q$ as a discount in response to the increased bargaining power of the buyers with an option to wait until period 2 .

As can be readily verified from (26) and as illustrated in Figure 2, the adjusted price

$$
\frac{\sigma_{f}^{1}}{1-k}=q
$$

is decreasing in the dependence parameter $k$. We can interpret this as the firms' response to the stronger incentive of the more interdependent consumers to delay their decisions.

Note that when the buyers are completely independent so that $k=0$, the equilibrium in Theorem 7 entails full delay since $x=0$ and $y=1 .^{15}$ On the other hand, when $k=0$, there exists another equilibrium with no delay as follows: The firms quote $\sigma^{1}=(1,1)$ in period 1, and all buyer types move in period 1: Type $s_{i}$ chooses $A$ if $s_{i}<\frac{1}{2}$ and $B$ if $s_{i}>\frac{1}{2}$. The conditional distribution $P_{i}(\cdot \mid h)$ when either buyer waits (i.e., after any $h \in H_{12}$ ) is the same as the prior (i.e., the uniform distribution over $[0,1]$ ). Since

\footnotetext{
${ }^{15}$ When the buyers discount the period 2 payoffs by $\delta<1$, we can show that the equilibrium with $k=0$ involves delay when $\delta$ is large. This equilibrium approaches the full delay equilibrium as $\delta \rightarrow 1$.
} 


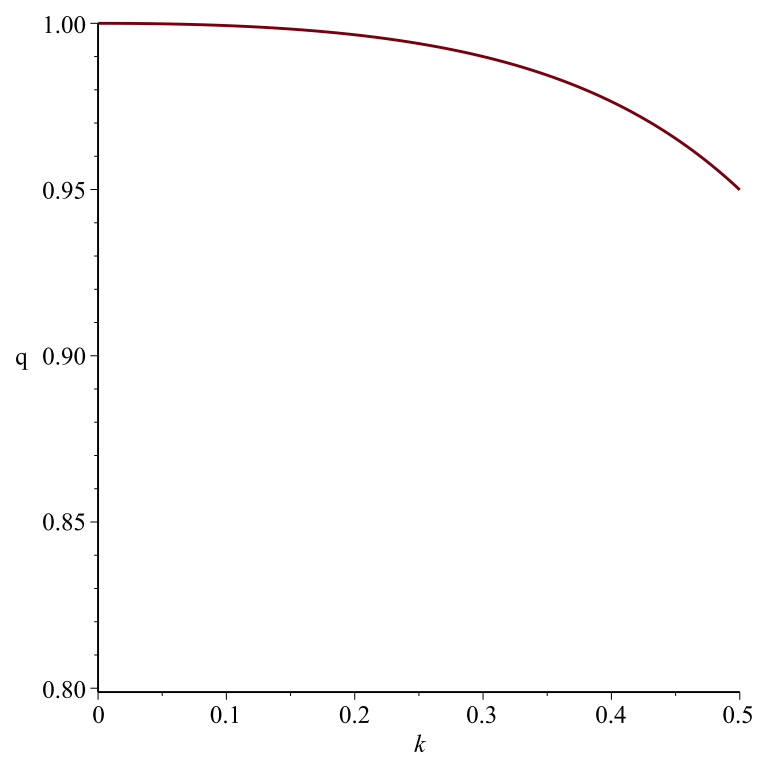

Figure 2: $q$ as a function of $k$.

the period 2 equilibrium price pair along any such history is again $(1,1)$, and since the buyers have no incentive to learn from the behavior of the other, their decision in period 1 not to wait is optimal. When $k>0$, however, there is no equilibrium of this type. If every buyer type moves in period 1 and if the price in period 2 is the same as that in period 1, then there exists a buyer type who has an incentive to wait and see. In other words, only the first equilibrium for $k=0$ is robust to a small perturbation in the value of $k$.

\section{Delay and Efficiency}

According to Theorem 7, the proportion of types who wait in period 1 is given by $1-x-(1-y)=q$, which is a decreasing function of $k$ as seen above. Hence, we have the following corollary.

Corollary 8. (Delay as a function of $k$ ) In the equilibrium of Theorem 7 , the probability of delay by either buyer equals $q$ and decreases as they become more interdependent.

Corollary 8 appears counter-intuitive since in general, the more interdependent is a buyer, the stronger is his incentive to learn from the behavior of the other buyer. In fact, we have seen in Proposition 5 that a higher degree of interdependence implies a 
larger delay when the period 2 price is not contingent on history. At first glance, it may seem that the smaller delay by a more interdependent buyer in Corollary 8 is caused by a lower adjusted price $\frac{\sigma_{f}^{1}}{1-k}=q$ in period 1 associated with a higher $k$. However, because of the martingale property (Lemma 4) and (14), the expected adjusted price in period 2 also equals

$$
\frac{1}{1-k} E\left[\sigma_{f}^{2}(h)\right]=1-2 x=q \quad \text { for } h \in H_{12},
$$

and is lowered by the same margin. Hence, the lower price in period 1 alone does not explain the decrease in delay in Corollary 8. Rather, the intuition is that in order to sustain the lower expected price in period 2 , the threshold value $x$ needs to be larger since $q$ and $x$ are inversely related as seen above: The larger is $x$, the smaller the interval of active buyer types in period 2 , and the more intense the competition between the firms. Since a higher $x$ is by definition equivalent to less delay, we have Corollary 8 .

We next turn to the (in)efficiency of the buyer decisions in equilibrium. First, the fully efficient outcome is obtained when the two buyers make their decisions after truthfully sharing private information about their types. Accordingly,

$$
\text { buyer } i \text { should choose } \begin{cases}A & \text { if } u_{i}>v_{i} \Leftrightarrow(1-k) s_{i}+k s_{j}<\frac{1}{2}, \\ B & \text { if } u_{i}<v_{i} \Leftrightarrow(1-k) s_{i}+k s_{j}>\frac{1}{2} .\end{cases}
$$

After some algebra, we can verify that the expected value of the ex post optimal decision is given by

$$
E\left[\max \left\{u_{i}, v_{i}\right\}\right]=E\left[\max \left\{1-(1-k) s_{i}-k s_{j},(1-k) s_{i}+k s_{j}\right\}\right]=\frac{3}{4} .
$$

In the equilibrium of Theorem 7, on the other hand,

$$
\text { buyer } i \text { chooses }\left\{\begin{aligned}
A \quad \text { if } \quad & s_{i}<c \text { and } s_{j}<x, \\
& s_{i}<\frac{1}{2} \text { and } s_{j} \in(x, 1-x), \\
& \text { or } s_{i}<1-c \text { and } s_{j}>1-x, \\
B \quad \text { if } \quad & s_{i}>c \text { and } s_{j}<x, \\
& s_{i}>\frac{1}{2} \text { and } s_{j} \in(x, 1-x), \\
& \text { or } s_{i}>1-c \text { and } s_{j}>1-x,
\end{aligned}\right.
$$

where $x=\frac{1-q}{2}$, and $c=\frac{2-k(1-q)}{12(1-k)}+\frac{1}{3}$ is the critical type of buyer $i$ that is indifferent between $A$ and $B$ in the period 2 market when $j$ chooses $A$ in period 1 (i.e., after $\left.h=\left(\sigma^{1}, \emptyset, A\right)\right)$. By symmetry, the critical type of $i$ indifferent between $A$ and $B$ in period 2 when $j$ chooses $B$ in period 1 is given by $1-c$. The ex post optimal and equilibrium 


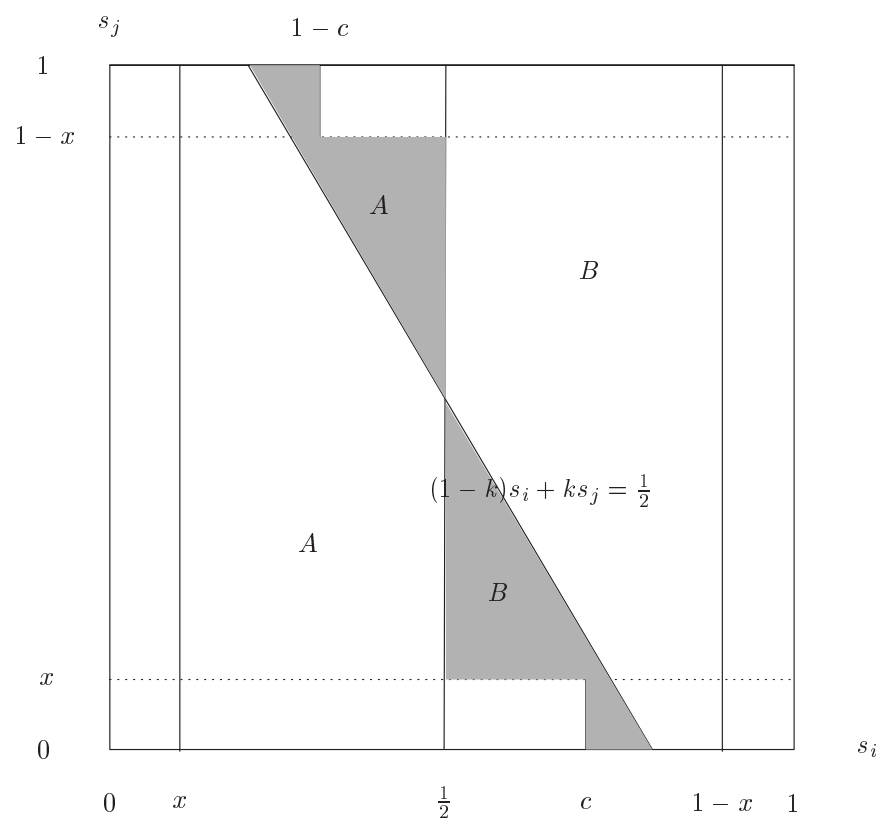

Figure 3: Optimal and equilibrium decisions by buyer $i$

decisions are illustrated in Figure 8. The shaded areas in the figure represent the signal realizations that lead to inefficient decisions. ${ }^{16}$

The expected value of the equilibrium decisions is hence given by

$$
\begin{aligned}
& \int_{0}^{x}\left\{\int_{0}^{c} v_{i} d s_{i}+\int_{c}^{1} w_{i} d s_{i}\right\} d s_{j}+\int_{1-x}^{1}\left\{\int_{0}^{1-c} v_{i} d s_{i}+\int_{1-c}^{1} w_{i} d s_{i}\right\} d s_{j} \\
&+\int_{x}^{1-x}\left\{\int_{0}^{\frac{1}{2}} v_{i} d s_{i}+\int_{\frac{1}{2}}^{1} w_{i} d s_{i}\right\} d s_{j} \\
&=2\left[c x+\frac{1}{2}(1-k)\left(1-2 c^{2}\right) x+\frac{k}{2}(1-2 c) x^{2}\right]+\frac{3-k}{4}(1-2 x) .
\end{aligned}
$$

As seen in Figure 4, the efficiency of the equilibrium buyer decisions is strictly decreasing in the dependence parameter $k$. This is expected from Corollary 8 since more interdependent buyers tend to move in period 1 more often.

\section{Discussions}

Throughout the paper, we have confined ourselves to the model with no discounting. An alternative interpretation of the no discounting assumption is that period 1 corresponds

\footnotetext{
${ }^{16}$ Indicated in the shaded areas are the (inefficient) equilibrium actions.
} 


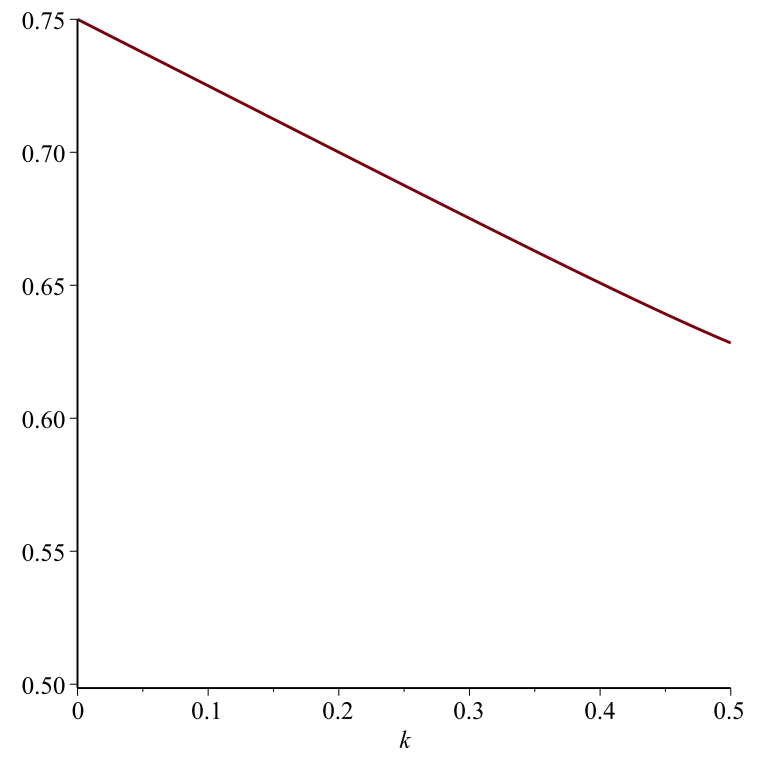

Figure 4: Equilibrium efficiency as a function of $k$.

to an advance sales period of the product. ${ }^{17}$ In this case, the consumption of the product takes place only after period 2 even if it is purchased in period 1 . When there is positive discounting, the most significant change takes place in the martingale property. It is then stated as the indifference condition for the critical type $x$ between choosing $A$ in period 1 and waiting and then unconditionally choosing $A$ in period 2, and the condition for type $y$ between $B$ in period 1 and the unconditional choice of $B$ in period 2. With this change, however, we expect the equilibrium under discounting to approach that under no discounting as the discount factor approaches one. We have not pursued this formally since the equation characterizing the equilibrium price as well as the specification of the off-equilibrium behavior is significantly more complex, and since this exercise does not appear to add new insights.

The assumption of the uniform distribution of the types is standard in the models of product differentiation and perhaps is the only one that admits analytical derivation of the equilibrium in our framework. While we admit that the assumption is restrictive in some ways, we also note that the specification of the distribution becomes less important when the degree of differentiation becomes small compared with the absolute values of the products as represented by the constant $u$ in the valuation function. Furthermore, our result suggests that problems with alternative distributions can be numerically analyzed

\footnotetext{
${ }^{17}$ See for example Yu et al. (2011).
} 
with the help of the martingale property.

Unlike in the majority of the social learning literature that assumes that a consumer's type $s_{i}$ is a noisy signal of the underlying state $\omega$, we have adopted an alternative framework in which there is no $\omega$ and the consumer types $s_{1}$ and $s_{2}$ are independent. In defense of our assumption, we should note that a few technical problems would arise under the alternative assumption of correlated signals. First, we would need to specify a family of conditional distributions of the signal for each state $\omega$. Specification of such conditional distributions is nontrivial and any specification would involve far more complicated analysis if possible at all. ${ }^{18}$ Second, if the firms do not know the realization of $\omega$, then we should consider the firms' incentive to learn $\omega$ through their pricing strategy. If they know $\omega$, on the other hand, we should think about their signaling incentives. Our assumption helps us abstract from these considerations, which could significantly complicate the problem.

In one interesting extension, we can consider a model in which the consumers are different in their interdependence levels. Targeting a particular class of consumers is shown to be a useful sales strategy in different contexts, and it would be interesting to examine if this is also the case in the present setting. ${ }^{19}$

\section{Appendix}

Proof of Lemma 2. Since the conditional probability $P_{i}(\cdot \mid h)$ of $s_{i}$ given $h \in H_{i}$ is the uniform distribution over the interval $(x, y)$, firm $A$ 's payoff from buyer $i$ in period 2 is explicitly given by:

$$
\pi_{A, i}^{2}\left(p^{2} \mid \tau_{i}^{2}, h\right)=\left\{\begin{aligned}
& \frac{p_{A}^{2}}{y-x}\left(\frac{1-2 k e_{j}(h)-p_{A}^{2}+p_{B}^{2}}{2(1-k)}-x\right) \\
& \quad \text { if } \frac{u+1-k e_{j}(h)-p_{A}^{2}}{1-k} \geq \frac{1-2 k e_{j}(h)-p_{A}^{2}+p_{B}^{2}}{2(1-k)} \in(x, y), \\
& \frac{p_{A}^{2}}{y-x}\left(\frac{u+1-k e_{j}(h)-p_{A}^{2}}{1-k}-x\right) \\
& \quad \text { if } \frac{1-2 k e_{j}(h)-p_{A}^{2}+p_{B}^{2}}{2(1-k)} \geq \frac{u+1-k e_{j}(h)-p_{A}^{2}}{1-k} \in(x, y), \\
& p_{A}^{2} \quad \text { if } \min \left\{\frac{1-2 k e_{j}(h)-p_{A}^{2}+p_{B}^{2}}{2(1-k)}, \frac{u+1-k e_{j}(h)-p_{A}^{2}}{1-k}\right\} \geq y, \\
& 0 \text { if } \min \left\{\frac{1-2 k e_{j}(h)-p_{A}^{2}+p_{B}^{2}}{2(1-k)}, \frac{u+1-k e_{j}(h)-p_{A}^{2}}{1-k}\right\} \leq x,
\end{aligned}\right.
$$

\footnotetext{
${ }^{18}$ One possibility is the binary specification of the signal. However, we have the problem of having no pure equilibrium in a stage game in this case.

${ }^{19}$ In a model where the dependence levels of consumers are observable to a monopolist seller, Aoyagi (2010) shows that it is optimal for the seller to target the least dependent consumers first and then move in the increasing order of the dependence levels.
} 
and firm $B$ 's payoff from buyer $i$ is given by:

$$
\pi_{B, i}^{2}\left(p^{2} \mid \tau^{2}, h\right)= \begin{cases}\frac{p_{B}^{2}}{y-x}\left(y-\frac{1-2 k e_{j}(h)-p_{A}^{2}+p_{B}^{2}}{2(1-k)}\right) \\ \quad \text { if } \frac{-u-k e_{j}(h)+p_{B}^{2}}{1-k} \leq \frac{1-2 k e_{j}(h)-p_{A}^{2}+p_{B}^{2}}{2(1-k)} \in(x, y), \\ \frac{p_{B}^{2}}{y-x}\left(y-\frac{-u-k e_{j}(h)+p_{B}^{2}}{1-k}\right) \\ \quad \text { if } \frac{1-2 k e_{j}(h)-p_{A}^{2}+p_{B}^{2}}{2(1-k)} \leq \frac{-u-k e_{j}(h)+p_{B}^{2}}{1-k} \in(x, y), \\ 0 & \text { if } \max \left\{\frac{1-2 k e_{j}(h)-p_{A}^{2}+p_{B}^{2}}{2(1-k)}, \frac{-u-k e_{j}(h)+p_{B}^{2}}{1-k}\right\} \geq y, \\ p_{B}^{2} & \text { if } \max \left\{\frac{1-2 k e_{j}(h)-p_{A}^{2}+p_{B}^{2}}{2(1-k)}, \frac{-u-k e_{j}(h)+p_{B}^{2}}{1-k}\right\} \leq x .\end{cases}
$$

We assume in the rest of the proof that $u>2(1-k)$ to avoid tedious case separation in the description of the best response that is immaterial to the description of the equilibrium. ${ }^{20}$

Let $R_{1}, \ldots, R_{4}$ be the sets of price profiles $\left(p_{A}^{2}, p_{B}^{2}\right)$ as illustrated in Figure 1. Explicitly, they are the set of $\left(p_{A}^{2}, p_{B}^{2}\right)$ satisfying $p_{A}^{2}, p_{B}^{2} \geq 0$, and

$$
\begin{aligned}
R_{1}: \quad & 1-2 k e_{j}(h)-2(1-k) y \leq p_{A}^{2}-p_{B}^{2} \leq 1-2 k e_{j}(h)-2(1-k) x, \\
& p_{A}^{2}+p_{B}^{2} \leq 2 u+1 \\
R_{2}: \quad & u+1-k e_{j}(h)-(1-k) y \leq p_{A}^{2} \leq u+1-k e_{j}(h)-(1-k) x, \\
& p_{A}^{2}+p_{B}^{2} \geq 2 u+1 \\
R_{3}: & p_{A}^{2}<u+1-k e_{j}(h)-(1-k) y, \\
& p_{A}^{2}-p_{B}^{2}<1-2 k e_{j}(h)-2(1-k) y ; \\
R_{4}: & p_{A}^{2}>\min \left\{p_{B}^{2}+1-2 k e_{j}(h)-2(1-k) x, u+1-k e_{j}(h)-(1-k) x\right\} .
\end{aligned}
$$

\footnotetext{
${ }^{20}$ This condition ensures that the intersection between $p_{A}^{2}-p_{B}^{2}=1-2 k e_{j}(h)-2(1-k) x$ and $p_{B}^{2}=$ $\frac{-1+2 k e_{j}(h)+2(1-k) y+p_{A}^{2}}{2}$ given by

$$
\left(p_{A}^{2}, p_{B}^{2}\right)=\left(1-2 k e_{j}(h)+2(1-k)(y-2 x), 2(1-k)(y-x)\right),
$$

and the intersection between $p_{A}^{2}-p_{B}^{2}=1-2 k e_{j}(h)-2(1-k) y$ and $p_{A}^{2}=\frac{1-2 k e_{j}(h)-2(1-k) x+p_{B}^{2}}{2}$ given by

$$
\left(p_{A}^{2}, p_{B}^{2}\right)=\left(2(1-k)(y-x),-1+2 k e_{j}(h)+2(1-k)(2 y-x)\right)
$$

are both below the participation constraint line $p_{A}^{2}+p_{B}^{2}=2 u+1$ so that the diagram is as depicted in Figure 1. The condition $u>1-k$ implied by this ensures that the maximum of $\pi_{A}^{2}\left(p^{2} \mid \tau_{i}^{2}, h\right)$ over $R_{2}$ is achieved at the left-end of the region at $p_{A}^{2}=u+1-k e_{j}(h)-(1-k) y$, and also that the maximum of $\pi_{A}^{2}\left(p^{2} \mid \tau_{i}^{2}, h\right)$ over the corresponding set is achieved at the lower-end of the region at $p_{B}^{2}=u+k e_{j}(h)+(1-k) x$ so that the best response functions are as described in Figure 1.
} 
We can express $\pi_{A, i}^{2}\left(p^{2} \mid \tau_{i}^{2}, h\right)$ in terms of these sets as

$$
\pi_{A, i}^{2}\left(p^{2} \mid \tau_{i}^{2}, h\right)= \begin{cases}\frac{p_{A}^{2}}{y-x}\left(\frac{1-2 k e_{j}(h)-p_{A}^{2}+p_{B}^{2}}{2(1-k)}-x\right) & \text { if }\left(p_{A}^{2}, p_{B}^{2}\right) \in R_{1}, \\ \frac{p_{A}^{2}}{y-x}\left(\frac{u+1-k e_{j}(h)-p_{A}^{2}}{1-k}-x\right) & \text { if }\left(p_{A}^{2}, p_{B}^{2}\right) \in R_{2}, \\ p_{A}^{2} & \text { if }\left(p_{A}^{2}, p_{B}^{2}\right) \in R_{3}, \\ 0 & \text { if }\left(p_{A}^{2}, p_{B}^{2}\right) \in R_{4} .\end{cases}
$$

It follows that firm $A$ 's period 2 best response correspondence is given by

$$
\operatorname{BR}_{A}\left(p_{B}^{2}\right)=\left\{\begin{array}{l}
\quad \text { if } 0 \leq p_{B}^{2}<\max \left\{0,-1+2 k e_{j}(h)+2(1-k) x\right\}, \\
\left\{\frac{1-2 k e_{j}(h)-2(1-k) x+p_{B}^{2}}{2}\right\} \\
\quad \text { if } p_{B}^{2} \geq \max \left\{0,-1+2 k e_{j}(h)+2(1-k) x\right\}, \text { and } \\
p_{B}^{2}<\max \left\{0,-1+2 k e_{j}(h)+2(1-k)(2 y-x)\right\}, \\
\left\{1-2 k e_{j}(h)-2(1-k) y+p_{B}^{2}\right\} \\
\text { if } p_{B}^{2} \geq \max \left\{0,-1+2 k e_{j}(h)+2(1-k)(2 y-x)\right\}, \text { and } \\
p_{B}^{2} \leq u+k e_{j}(h)+(1-k) y, \\
\left\{u+1-k e_{j}(h)-(1-k) y\right\} \quad \text { if } p_{B}^{2}>u+k e_{j}(h)+(1-k) y .
\end{array}\right.
$$

Likewise, firm B's period 2 best response correspondence is given by

$$
\mathrm{BR}_{B}\left(p_{A}^{2}\right)=\left\{\begin{array}{l}
\quad \begin{array}{l}
\boldsymbol{R}_{+} \quad \text { if } 0 \leq p_{A}^{2}<\max \left\{1-2 k e_{j}(h)-2(1-k) y, 0\right\}, \\
\left\{\frac{-1+2 k e_{j}(h)+2(1-k) y+p_{A}^{2}}{2}\right\} \\
\text { if } p_{A}^{2} \geq \max \left\{1-2 k e_{j}(h)-2(1-k) y, 0\right\}, \text { and }
\end{array} \\
p_{A}^{2}<\max \left\{0,1-2 k e_{j}(h)-2(1-k)(2 x-y)\right\}, \\
\left\{1-2 k e_{j}-2(1-k) x+p_{A}^{2}\right\} \\
\text { if } p_{A}^{2} \geq \max \left\{0,1-2 k e_{j}(h)-2(1-k)(2 x-y)\right\}, \text { and } \\
p_{A}^{2}<u+1-k e_{j}(h)-(1-k) x, \\
\left\{u+k e_{j}(h)+(1-k) x\right\} \quad \text { if } p_{B}^{2} \geq u+1-k e_{j}(h)-(1-k) x .
\end{array}\right.
$$

Figure 1 depicts these best response correspondences for the case $2(1-k) x<1-$ $2 k e_{j}(h)<2(1-k) y$. Note also that when $p_{A}^{2}-p_{B}^{2} \leq 1-2 k e_{j}(h)-2(1-k) y$, firm $A$ monopolizes the market under $\left(p_{A}^{2}, p_{B}^{2}\right)$, and that when $p_{A}^{2}-p_{B}^{2} \geq 1-2 k e_{j}(h)-2(1-$ $k) x$, firm $B$ monopolizes the market under $\left(p_{A}^{2}, p_{B}^{2}\right)$. Note also that the participation constraint does not bind for the critical type that is indifferent between firms $A$ and $B$ if $p_{A}^{2}+p_{B}^{2}<2 u+1$. 
a) $1-2 k e_{j}(h) \in[2(1-k)(2 x-y), 2(1-k)(2 y-x)]$.

The best response correspondences $p_{A}^{2}=\mathrm{BR}_{A}\left(p_{B}^{2}\right)$ and $p_{B}^{2}=\mathrm{BR}_{B}\left(p_{A}^{2}\right)$ have a unique intersection

$$
\left(\frac{1-2 k e_{j}(h)+2(1-k)(y-2 x)}{3}, \frac{-1+2 k e_{j}(h)+2(1-k)(2 y-x)}{3}\right),
$$

which satisfies $1-2 k e_{j}(h)-2(1-k) x<p_{A}^{2}-p_{B}^{2}<1-2 k e_{j}(h)-2(1-k) y$ and also $p_{A}^{2}+p_{B}^{2}<2 u+1$ when $u>\frac{1}{2}-k$. Hence, the two firms segment the market and the critical type is given by $s_{i}=\frac{1-2 k e_{j}(h)}{6(1-k)}+\frac{x+y}{3}$.

b) $1-2 k e_{j}(h)>2(1-k)(2 y-x)$.

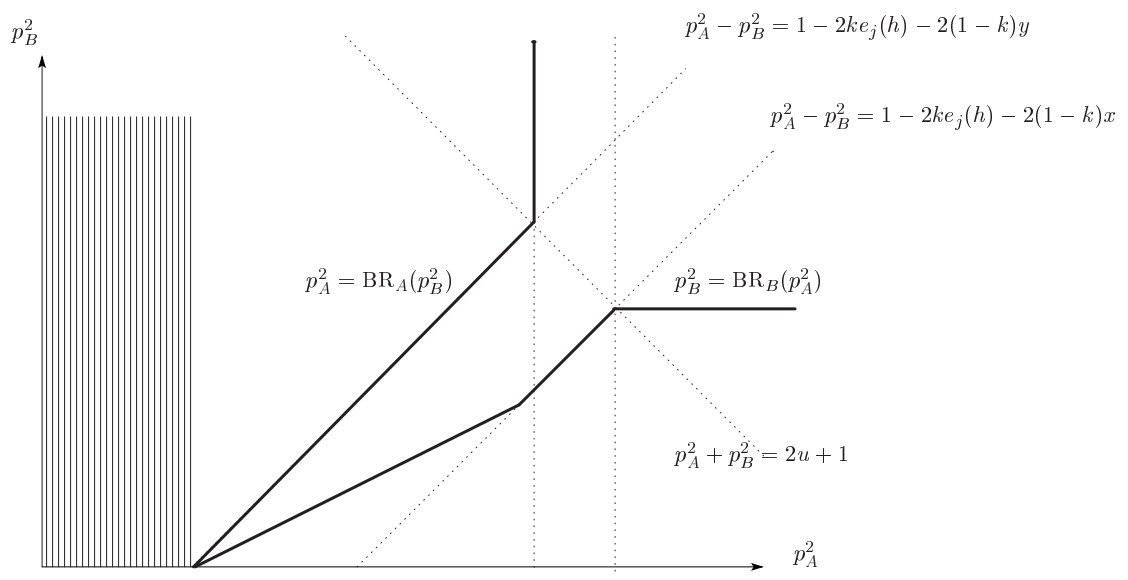

Figure 5: Best-response diagram: $A$-corner equilibrium

As seen in Figure 5, the unique fixed point of the joint best-response correspondence $\left(p_{A}^{2}, p_{B}^{2}\right) \rightarrow\left(\mathrm{BR}_{A}\left(p_{B}^{2}\right), \mathrm{BR}_{B}\left(p_{A}^{2}\right)\right)$ is given by

$$
\left(1-2 k e_{j}(h)-2(1-k) y, 0\right) .
$$

Since $p_{A}^{2}-p_{B}^{2}=1-2 k e_{j}(h)-2(1-k) y$, firm $A$ monopolizes the market.

c) $1-2 k e_{j}(h)<2(1-k)(2 x-y)$.

As in the previous case, the unique fixed point of the joint best-response correspondence $\left(p_{A}^{2}, p_{B}^{2}\right) \rightarrow\left(\mathrm{BR}_{A}\left(p_{B}^{2}\right), \mathrm{BR}_{B}\left(p_{A}^{2}\right)\right)$ is given by

$$
\left(0,-1+2 k e_{j}(h)+2(1-k) x\right) .
$$

Since $p_{A}^{2}-p_{B}^{2} \geq 1-2 k e_{j}(h)-2(1-k) x$, firm $B$ monopolizes the market.

This completes the proof. 
Proof of Lemma 1. We will show that if $\tau_{i}^{1}\left(s_{i}, p^{1}\right)=A$ for some $s_{i}$ and $s_{i}^{\prime}<s_{i}$, then $\tau_{i}\left(s_{i}^{\prime}, p^{1}\right)=A$. By setting $x\left(p^{1}\right)=\sup \left\{s_{i}: \tau_{i}^{1}\left(s_{i}, p^{1}\right)=A\right\}$, it would then follow that $\tau_{i}^{1}\left(s_{i}, p^{1}\right)=A$ if $s_{i}<x$.

Suppose that $\tau_{i}^{1}\left(s_{i}, p^{1}\right)=A$ and that $s_{i}^{\prime}<s_{i}$. Since type $s_{i}$ prefers choosing $A$ to choosing $B$ in period 1 , we have

$$
E_{s_{j}}\left[v_{i} \mid s_{i}\right]-p_{A}^{1} \geq E_{s_{j}}\left[w_{i} \mid s_{i}\right]-p_{B}^{1} .
$$

Likewise, since type $s_{i}$ prefers choosing $A$ in period 1 to waiting and then choosing either $A, \emptyset$, or $B$ in period 2 , we also have

$$
E_{s_{j}}\left[v_{i} \mid s_{i}\right]-p_{A}^{1} \geq \delta \sum_{h \in H_{i}} P(h) \max \left\{E_{s_{j}}\left[v_{i} \mid s_{i}, h\right]-\sigma_{A}^{2}(h), 0, E_{s_{j}}\left[w_{i} \mid s_{i}, h\right]-\sigma_{B}^{2}(h)\right\},
$$

where $H_{i}\left(p^{1}\right)=\left\{h=\left(p^{1}, d^{1}\right): d_{i}^{1}=\emptyset\right\}$ is the set of period 1 histories along which the firms quote $p^{1}$ and buyer $i$ chooses to wait. Note now that

$$
\begin{aligned}
& E_{s_{j}}\left[v_{i} \mid s_{i}^{\prime}\right]=(1-k)\left(s_{i}-s_{i}^{\prime}\right)+E_{s_{j}}\left[v_{i} \mid s_{i}\right]>E_{s_{j}}\left[v_{i} \mid s_{i}\right], \quad \text { and } \\
& E_{s_{j}}\left[w_{i} \mid s_{i}^{\prime}\right]=-(1-k)\left(s_{i}-s_{i}^{\prime}\right)+E_{s_{j}}\left[w_{i} \mid s_{i}\right]<E_{s_{j}}\left[w_{i} \mid s_{i}\right] .
\end{aligned}
$$

It then immediately follows that (21) holds for type $s_{i}^{\prime}$ so that it strictly prefers choosing $A$ to choosing $B$ in period 1 . To see that $s_{i}^{\prime}$ also prefers choosing $A$ to waiting, add $(1-k)\left(s_{i}-s_{i}^{\prime}\right)$ to both sides of $(22)$. We then have

$$
\begin{aligned}
& E_{s_{j}}\left[v_{i} \mid s_{i}^{\prime}\right]-p_{A}^{1} \\
& \geq(1-\delta)(1-k)\left(s_{i}-s_{i}^{\prime}\right) \\
& +\delta \sum_{h \in H_{i}} P(h) \max \left\{E_{s_{j}}\left[v_{i} \mid s_{i}^{\prime}, h\right]-\sigma_{A}^{2}(h),(1-k)\left(s_{i}-s_{i}^{\prime}\right),\right. \\
& \left.\quad(1-k)\left(s_{i}-s_{i}^{\prime}\right)+E_{s_{j}}\left[w_{i} \mid s_{i}, h\right]-\sigma_{B}^{2}(h)\right\} \\
& >\delta \sum_{h \in H_{i}} P(h) \max \left\{E_{s_{j}}\left[v_{i} \mid s_{i}^{\prime}, h\right]-\sigma_{A}^{2}(h), 0, E_{s_{j}}\left[w_{i} \mid s_{i}^{\prime}, h\right]-\sigma_{B}^{2}(h)\right\},
\end{aligned}
$$

which shows that (22) holds for type $s_{i}^{\prime}$ with strict inequality, and hence it strictly prefers choosing $A$ to waiting. It can be shown similarly that there exists $y$ such that $\tau_{i}^{1}\left(s_{i}, p^{1}\right)=B$ if $s_{i}>y$. If $s_{i} \in(x, y)$, then we cannot have $\tau_{i}^{1}\left(s_{i}, p^{1}\right)=A$ since that would imply $\tau_{i}^{1}\left(s_{i}^{\prime}, p^{1}\right)=A$ for some $s_{i}^{\prime}>x$, a contradiction. Since we cannot have $\tau_{i}^{1}\left(s_{i}, p^{1}\right)=B$ either, we must have $\tau_{i}^{1}\left(s_{i}, p^{1}\right)=\emptyset$.

Proof of Lemma 4. We first show that if $\sigma^{2}(h)$ is as given by Lemma 2, then after any $d_{j}^{1}$, type $x$ 's payoff from unconditionally choosing $A$ in period 2 equals that from following 
the sequentially rational strategy $\tau_{i}^{2}: E_{s_{j}}\left[\pi_{i}^{2}\left(x, s_{j}, \sigma^{2}(h), \tau_{i}^{2}\left(x, h, \sigma^{2}(h)\right) \mid s_{i}, h\right]\right.$, where $h=\left(p^{1}, \emptyset, d_{j}^{1}\right)$. For this, note that type $x$ is the lowest type in the period 2 market. Hence, after any decision $d_{j}^{1}$ of buyer $j$, if $d^{1}=\left(\emptyset, d_{j}^{1}\right)$ is followed by an interior equilibrium or an $A$-corner equilibrium (Lemma 2), then type $x$ will optimally choose $A$ in period 2 after $d^{1}$. On the other hand, if $d^{1}$ is followed by a $B$-corner equilibrium, then type $x$ is just indifferent between $A$ and $B$ after $h=\left(p^{1}, d^{1}\right)$ : When $1-2 k e_{j}(h)<2(1-k)(2 x-y)$, (7) implies that

type $x$ 's payoff from choosing $A$

$$
\begin{aligned}
& =u+1-(1-k) x-k e_{j}(h)-0 \\
& =u+(1-k) x+k e_{j}(h)-\left(-1+2 k e_{j}(h)+2(1-k) x\right) \\
& =\text { type } x \text { 's payoff from choosing } B .
\end{aligned}
$$

It follows that in period 2, choosing $A$ unconditionally is optimal for type $x$ regardless of buyer $j$ 's decision $d_{j}^{1}$ or the type of equilibrium that follows $d^{1}$. This in turn implies that type $x$ 's payoff from waiting in period 1 equals that from waiting and then unconditionally choosing $A$ in period 2 . Now in period 1 , if $x>0$ and waiting is strictly better than choosing $A$, then for $\epsilon>0$ small, type $s_{i}=x-\epsilon>0$ also finds it strictly better off waiting, which is a contradiction to the sequential rationality of $\tau_{i}^{1}$. On the other hand, if $x<1$ and choosing $A$ in period 1 is strictly better than waiting, then for $\epsilon>0$ small, type $s_{i}=x+\epsilon<1$ finds it strictly better off choosing $A$ in period 1, which is again a contradiction to the sequential rationality of $\tau_{i}^{1}$. Hence, type $s_{i}=x$ is indifferent between choosing $A$ and waiting in period 1. Combining the two observations together, we have

$$
E\left[v_{i} \mid s_{i}=x\right]-p_{A}^{1}=E\left[E_{s_{j}}\left[v_{i} \mid s_{i}=x, h\right]-\sigma_{A}^{2}(h) \mid s_{i}=x\right],
$$

where the left-hand side is buyer $i$ 's payoff from buying $A$ in period 1 , and the righthand side is his payoff from waiting and then unconditionally choosing $A$ in period 2 . However, since we have by the law of iterated expectations

$$
E\left[v_{i} \mid s_{i}=x\right]=E\left[E_{s_{j}}\left[v_{i} \mid s_{i}=x, h\right] \mid s_{i}=x\right],
$$

the above implies that

$$
p_{A}^{1}=E\left[\sigma_{A}^{2}\left(p^{1}, \emptyset, d_{j}^{1}\right)\right] .
$$

The symmetric discussion proves the statement for the price of $B$. 
Proof of Proposition 6. Define $q=\frac{\sigma_{1}^{A}}{1-k}=\frac{\sigma_{B}^{1}}{1-k}$ to be the adjusted period 1 price in equilibrium. It follows from (12) that the range of $q$ is given by

$$
q \in\left[\frac{k}{6-7 k}, \frac{k}{4(1-k)}\right] \text {. }
$$

Firm $A$ 's payoff over two periods under $(q, q)$ is given by

$$
\begin{aligned}
& \hat{\Pi}_{A}(q, q) \\
& =(1-k) q x^{*}+\left(1-2 x^{*}\right)\left[x^{*} \pi_{A}^{2 *}\left(\sigma^{1}, \emptyset, A\right)+\left(1-2 x^{*}\right) \pi_{A}^{2 *}\left(\sigma^{1}, \emptyset, \emptyset\right)\right] \\
& =x\left\{(6-7 k)\left(x^{*}\right)^{2}-(5-6 k) x^{*}+1-k\right\} \\
& +\left(1-2 x^{*}\right) x^{*}\left\{1-k x^{*}-2(1-k)\left(1-x^{*}\right)\right\} \\
& +\left(1-2 x^{*}\right) \frac{\left\{1-k+2(1-k)\left(1-2 x^{*}\right)\right\}^{2}}{18(1-k)} \\
& =(-2+3 k)\left(x^{*}\right)^{3}+(5-7 k)\left(x^{*}\right)^{2}+(-3+4 k) x^{*}+\frac{1-k}{2} \\
& \equiv \varphi\left(x^{*}\right) .
\end{aligned}
$$

Since $\varphi$ is convex over $\left[0, \frac{5-7 k}{3(2-3 k)}\right]$, and since $0<\frac{2}{5}<\frac{3-4 k}{6-7 k}<\frac{1}{2}<\frac{5-7 k}{3(2-3 k)}$, for $x^{*}$ satisfying (10), we have

$$
\begin{aligned}
\varphi\left(x^{*}\right) & \leq \max \left\{\varphi\left(\frac{1}{2}\right), \varphi\left(\frac{2}{5}\right)\right\} \\
& =\max \left\{\frac{k}{8}, \frac{-7+43 k}{250}\right\}=\frac{k}{8} .
\end{aligned}
$$

Consider now firm $A$ 's deviation from $\sigma_{A}^{1}$ to $p_{A}^{1}>u+1$. Define $q_{A}=\frac{p_{A}^{1}}{1-k}, x=x\left(p_{A}^{1}, \sigma_{B}^{1}\right)$ and $y=y\left(p_{A}^{1}, \sigma_{B}^{1}\right)$. It is clear that no buyer chooses $A$ in period 1 and hence $x=0$. On the other hand, the martingale property (Lemma 4$)$ under the price pair $\left(p_{A}^{1},(1-k) q\right)$ implies that

$$
(1-k) q=y \frac{-1+k y+2(1-k) \cdot 2 y}{3}+(1-y) \frac{-1+k(1+y)+2(1-k) \cdot 2 y}{3},
$$

or equivalently,

$$
y=\frac{1+3 q}{4} .
$$

Firm $A$ 's payoff over the two periods under $\left(q_{A}, q\right)$ is given by

$$
\begin{aligned}
\hat{\Pi}_{A}\left(q_{A}, q\right) & =y \frac{\{1-k y+2(1-k) y\}^{2}}{18(1-k)}+(1-y) \frac{\{1-k(1+y)+2(1-k) y\}^{2}}{18(1-k)} \\
& =\frac{1}{18(1-k)}\left[\left\{4(1-k)^{2}-k^{2}\right\} y^{2}+\left\{4(1-k)^{2}+k^{2}\right\} y+(1-k)^{2}\right] .
\end{aligned}
$$


Since $y>\frac{1}{4}$ by (24), we have

$$
\hat{\Pi}_{A}\left(q_{A}, q\right)>\frac{1}{18(1-k)}\left[\frac{9}{4}(1-k)^{2}+\frac{3}{16} k^{2}\right] \geq \frac{1}{8}(1-k) .
$$

It follows from (9) and (25) that for any $q$ satisfying (23),

$$
\hat{\Pi}_{A}\left(q_{A}, q\right)>\hat{\Pi}_{A}(q, q) .
$$

Hence, $\sigma_{A}^{1}=(1-k) q$ cannot be an equilibrium price for firm $A$.

Proof of Theorem 7. Consider the following pair of a strategy profile $(\sigma, \tau)$ and conditional beliefs $P_{i}(\cdot \mid h)$ is a symmetric equilibrium of the two-period model.

- Period 1 strategies:

For $q$ given in (26), firm $f$ quotes

$$
\sigma_{f}^{1}=(1-k) q,
$$

and for any $p^{1}=\left(p_{A}^{1}, p_{B}^{1}\right)$ and $\left(q_{A}, q_{B}\right)=\left(\frac{p_{A}^{1}}{1-k}, \frac{p_{B}^{1}}{1-k}\right)$, buyer $i$ 's decision is given by (3) for $x$ and $y$ defined as follows:

1. If $\max \left\{q_{A}, q_{B}\right\}>\frac{3-2 k}{6(1-k)}, 2 q_{A}-q_{B} \leq 1$, and $2 q_{B}-q_{A} \leq 1$, then

$$
x=\frac{1-2 q_{A}+q_{B}}{2} \text { and } y=\frac{1+2 q_{B}-q_{A}}{2} .
$$

2. If $\frac{k}{4-3 k} \leq q_{A} \leq 1$ and $q_{B} \geq \max \left\{\frac{3-2 k}{6(1-k)}, \frac{q_{A}+1}{2}\right\}$, then

$$
x=\frac{3\left(1-q_{A}\right)}{4} \quad \text { and } \quad y=1 .
$$

3. If $q_{A}<\frac{k}{4-3 k}$ and $q_{B} \geq \frac{3-2 k}{6(1-k)}$, then

$$
x=\frac{3-2 k+\sqrt{(3-2 k)^{2}-12(4-3 k)(1-k) q_{A}}}{2(4-3 k)} \quad \text { and } \quad y=1 .
$$

4. If $\frac{k}{4-3 k} \leq q_{B} \leq 1$ and $q_{A} \geq \max \left\{\frac{3-2 k}{6(1-k)}, \frac{q_{B}+1}{2}\right\}$, then

$$
x=0 \quad \text { and } \quad y=1-\frac{3\left(1-q_{B}\right)}{4} .
$$


5. If $q_{B}<\frac{k}{4-3 k}$ and $q_{A} \geq \frac{3-2 k}{6(1-k)}$, then

$$
x=0 \quad \text { and } \quad y=1-\frac{3-2 k+\sqrt{(3-2 k)^{2}-12(4-3 k)(1-k) q_{B}}}{2(4-3 k)} .
$$

6. If $\min \left\{q_{A}, q_{B}\right\}>1$, then

$$
x=0 \quad \text { and } \quad y=1 \text {. }
$$

7. If $\max \left\{q_{A}, q_{B}\right\}<\frac{3-2 k}{6(1-k)}$, then

$$
x=y=\frac{1-q_{A}+q_{B}}{2} .
$$

- Beliefs:

The conditional distribution $P_{i}(\cdot \mid h)$ about buyer $i$ 's type $s_{i}$ given history $h=\left(p^{1}, d^{1}\right)$ is derived through Bayes' rule if buyer $i$ chooses $d_{i}^{1}$ with positive probability when faced with $p^{1}: P\left(\tau_{i}^{1}\left(s_{i}, p^{1}\right)=d_{i}^{1}\right)>0$. Otherwise, $P_{i}(\cdot \mid h)$ equals the prior and is given by the uniform distribution over $[0,1]$.

- Period 2 strategies:

Let $e_{j}(h)=E_{s_{j}}\left[s_{j} \mid h\right]$ denote the expected value of $s_{j}$ according to the conditional distribution $P_{s_{j}}(\cdot \mid h)$ specified above. Then the firms' strategy profile $\sigma^{2}$ in period 2 is given as in Lemma 2, and each buyer's strategy is given by (2).

Figure 6 illustrates the classification of the period 1 price pair $\left(q_{A}, q_{B}\right)=\left(\frac{p_{A}^{1}}{1-k}, \frac{p_{B}^{1}}{1-k}\right)$ in Theorem 7. Note that the equilibrium price pair in period 1 belongs to $R_{1}$.

It is clear from the discussion in the preceding section that the period 2 strategies of the firms and buyers are optimal. In what follows, we first show that the period 1 strategies of the buyers are optimal, and then show that the firms' period 1 price quote (26) is also optimal given the buyers' strategies. In what follows, given any price pair $p^{1}$ and decision pair $d^{1}$ in period 1 , let $p_{f}^{2 *}\left(d^{1}\right)=\sigma_{f}^{2}\left(p^{1}, d^{1}\right)$ denote the price quoted by firm $f$ after history $h=\left(p^{1}, d^{1}\right)$.

Step 1. We first examine the optimality of the buyers' period 1 strategies for each period 1 price profile as classified in Figure 6 .

1. $\left(q_{A}, q_{B}\right) \in R_{1}: \max \left\{q_{A}, q_{B}\right\}>\frac{3-2 k}{6(1-k)}, 2 q_{A}-q_{B} \leq 1$, and $2 q_{B}-q_{A} \leq 1$.

Substituting

$$
x=\frac{1-2 q_{A}+q_{B}}{2} \text { and } \quad y=\frac{1+2 q_{B}-q_{A}}{2}
$$




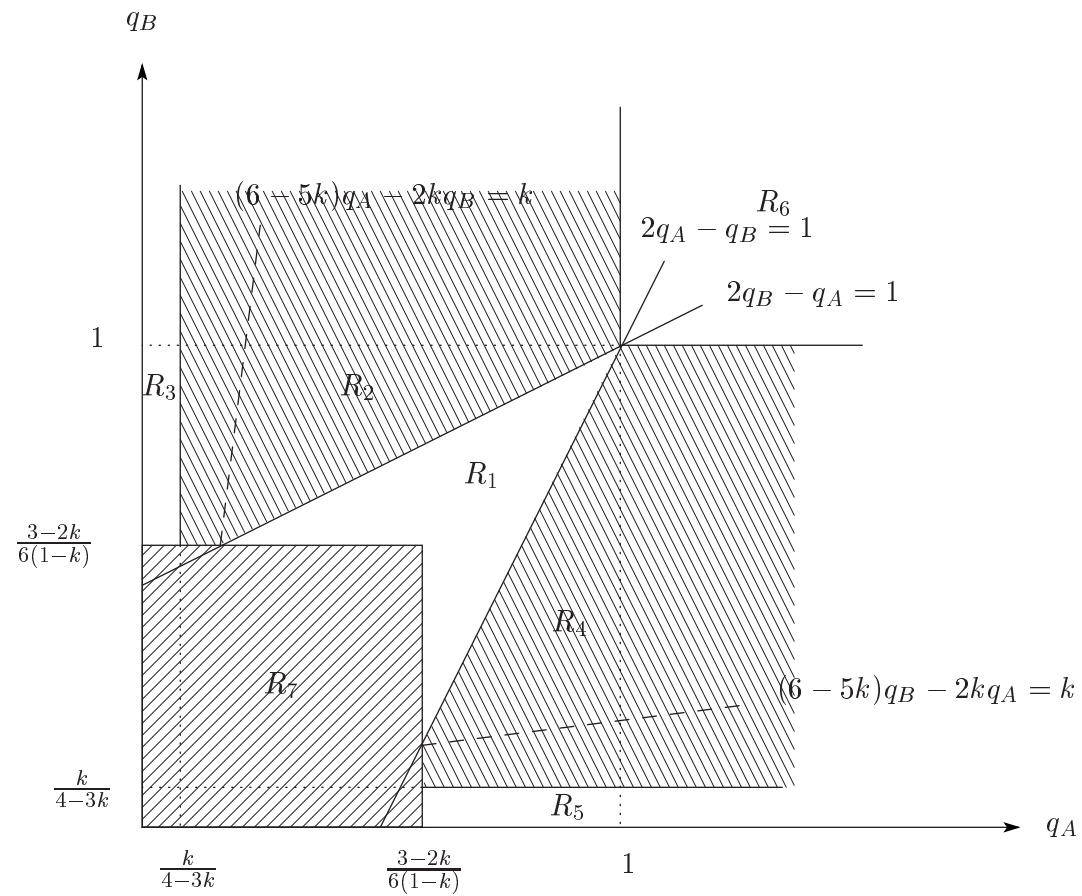

Figure 6: Classification of the period 1 price pair $\left(q_{A}, q_{B}\right)$.

into the conditions (13) ensuring the interior equilibrium in period 2 after every $d^{1}$ (i.e., $d^{1}=(\emptyset, A),(\emptyset, \emptyset)$, and $\left.(\emptyset, B)\right)$, we obtain

$$
(6-5 k) q_{A}-2 k q_{B} \geq k, \quad \text { and } \quad(6-5 k) q_{B}-2 k q_{A} \geq k .
$$

As is clear from Figure $6,\left(q_{A}, q_{B}\right)$ under consideration satisfies these conditions. The period 2 equilibrium prices are then given by (6) for each $d^{1}$, and the expected period 2 price is given by (14).

Note from (15) and (16) that when $\delta=1,(x, y)$ is chosen so that the period 1 price of good $f$ equals its expected period 2 price. In other words, for $f=A$ and $B$,

$$
p_{f}^{1}=x p_{f}^{2 *}(\emptyset, A)+(y-x) p_{f}^{2 *}(\emptyset, \emptyset)+(1-y) p_{f}^{2 *}(\emptyset, B) .
$$

We will now examine buyer $i$ 's incentive depending on his type $s_{i}$. Note first that the following inequalities hold under (27):

$$
x \leq \frac{1-k(1+y)}{6(1-k)}+\frac{x+y}{3}<\frac{1-k(x+y)}{6(1-k)}+\frac{x+y}{3}<\frac{1-k x}{6(1-k)}+\frac{x+y}{3} \leq y .
$$

In the above, $s_{i}<x$ implies that $s_{i}$ chooses $A$ in period 1 , and $s_{i}>y$ implies that $s_{i}$ chooses $B$ in period 1 . On the other hand, Lemma 2 implies that the three 
quantities in the middle are the critical types $s_{i}$ of buyer $i$ who are indifferent between $A$ and $B$ in period 2 after buyer $j$ 's choice of $d_{j}^{1}=B, \emptyset$ and $A$ in period 1 , respectively. It follows that there are the following six cases to consider depending on buyer $i$ 's decision over two periods.

- Type $s_{i}$ chooses $A$ in period $1 \Leftrightarrow s_{i}<x$.

It follows from (28) that any such type, if forced to wait in period 1, would choose $A$ in period 2 regardless of buyer $j$ 's decision in period 1 . If type $s_{i}$ chooses $A$ in period 1 , his expected payoff equals

$$
E\left[v_{i} \mid s_{i}\right]-p_{A}^{1}
$$

By Lemma 4, however, the above is also type $s_{i}$ 's expected payoff from waiting and then choosing $A$ after any $d_{j}^{1}$. Hence, choosing $A$ in period 1 is just as good as waiting, and is optimal.

- Type $s_{i}$ waits in period 1 and then chooses $A$ in period 2 regardless of buyer $j$ 's decision $d_{j}^{1}$ : $s_{i} \in\left(x, \frac{1-k(1+y)}{6(1-k)}+\frac{x+y}{3}\right)$.

Any such type $s_{i}$ prefers $A$ to $B$ if forced to move in period 1 since

$$
\frac{1-q_{A}+q_{B}}{2}=\frac{1}{6}+\frac{x+y}{3}<\frac{1-k(1+y)}{6(1-k)}+\frac{x+y}{3} .
$$

It then follows from the same logic as above that any such type is indifferent between choosing $A$ and waiting in period 1. Hence, waiting is optimal for $s_{i}$.

- Type $s_{i}$ waits in period 1 and then chooses $A$ if $d_{j}^{1}=A$ or $\emptyset$, and $B$ if $d_{j}^{1}=B$ : $s_{i} \in\left(\frac{1-k(1+y)}{6(1-k)}+\frac{x+y}{3}, \frac{1-k(x+y)}{6(1-k)}+\frac{x+y}{3}\right)$.

Let $p_{A}^{2 *}\left(d^{1}\right)=\sigma_{A}^{2}\left(p^{1}, d^{1}\right)$. Then type $s_{i}$ 's decision in period 2 implies:

$$
\begin{aligned}
& E_{s_{j}}\left[v_{i} \mid p^{1}, d_{j}^{1}=A\right]-p_{A}^{2 *}(\emptyset, A) \geq E_{s_{j}}\left[w_{i} \mid p^{1}, d_{j}^{1}=A\right]-p_{B}^{2 *}(\emptyset, A), \\
& E_{s_{j}}\left[v_{i} \mid p^{1}, d_{j}^{1}=\emptyset\right]-p_{A}^{2 *}(\emptyset, \emptyset) \geq E_{s_{j}}\left[w_{i} \mid p^{1}, d_{j}^{1}=\emptyset\right]-p_{B}^{2 *}(\emptyset, \emptyset), \\
& E_{s_{j}}\left[w_{i} \mid p^{1}, d_{j}^{1}=B\right]-p_{B}^{2 *}(\emptyset, B) \geq E_{s_{j}}\left[v_{i} \mid p^{1}, d_{j}^{1}=B\right]-p_{A}^{2 *}(\emptyset, B) .
\end{aligned}
$$

Hence, type $s_{i}$ 's payoff from waiting is greater than or equal to his payoff from choosing $A$ after any $d_{j}^{1}$ or choosing $B$ after any $d_{j}^{1}$. Since his payoff from choosing $A$ in period 1 equals that from waiting and then choosing $A$ after any $d_{j}^{1}$ (Lemma 4 ), and likewise his payoff from choosing $B$ in period 1 equals that from waiting and then choosing $B$ after any $d_{j}^{1}$, we see that waiting in period 1 is optimal for any such type $s_{i}$. 
- Type $s_{i}$ waits in period 1 and then chooses $A$ if $d_{j}^{1}=A$ and $B$ if $\emptyset$ or $d_{j}^{1}=B$ : $s_{i} \in\left(\frac{1-k(x+y)}{6(1-k)}+\frac{x+y}{3}, \frac{1-k x}{6(1-k)}+\frac{x+y}{3}\right)$.

By the same logic as above, type $s_{i}$ 's payoff from waiting in period 1 is greater than or equal to choosing either $A$ or $B$ in period 1 .

- Type $s_{i}$ waits in period 1 and then chooses $B$ after any $d_{j}^{1}: s_{i} \in\left(\frac{1-k x}{6(1-k)}+\frac{x+y}{3}, y\right)$.

- Type $s_{i}$ chooses $B$ in period 1: $s_{i}>y$.

By the same logic as in the first two cases, choosing $B$ and waiting in period 1 are both optimal for any type in these two intervals.

2. $\left(q_{A}, q_{B}\right) \in R_{2}: \frac{k}{4-3 k} \leq q_{A} \leq 1$ and $q_{B} \geq \max \left\{\frac{3-2 k}{6(1-k)}, \frac{q_{A}+1}{2}\right\}$.

Since $y=1, d_{j}^{1}=B$ occurs with probability zero. We can see that when

$$
x=\frac{3\left(1-q_{A}\right)}{4} \quad \text { and } \quad y=1,
$$

$q_{A} \geq \frac{k}{4-3 k}$ is equivalent to the condition

$$
1-k(x+y) \geq 2(1-k)(2 x-y)
$$

which ensures the interior equilibrium in period 2 after $d^{1}=(\emptyset, A)$ and $(\emptyset, \emptyset)$. Furthermore, $q_{A} \geq \frac{k}{4-3 k}$ also implies

$$
x \leq \frac{1-k(x+1)}{6(1-k)}+\frac{x+1}{3}<\frac{1-k x}{6(1-k)}+\frac{x+1}{3} \leq 1 .
$$

The two quantities in the middle are the critical types of buyer $i$ who are indifferent between $A$ and $B$ in period 2 after $d_{j}^{1}=\emptyset$ and $d_{j}^{1}=A$, respectively. Therefore, we need to check the optimality of $i$ 's behavior in the following four cases.

- Type $s_{i}$ chooses $A$ in period 1: $s_{i}<x$.

- Type $s_{i}$ waits in period 1 and then chooses $A$ after $d_{j}^{1}=A$ or $\emptyset: s_{i} \in$ $\left(x, \frac{1-k(x+1)}{6(1-k)}+\frac{x+1}{3}\right)$.

- Type $s_{i}$ waits in period 1 and then chooses $A$ after $d_{j}^{1}=A$ and chooses $B$ after $d_{j}^{1}=\emptyset: s_{i} \in\left(\frac{1-k(x+1)}{6(1-k)}+\frac{x+1}{3}, \frac{1-k x}{6(1-k)}+\frac{x+1}{3}\right)$.

- Type $s_{i}$ waits in period 1 and then chooses $B$ after $d_{j}^{1}=A$ or $\emptyset: s_{i}>$ $\frac{1-k x}{6(1-k)}+\frac{x+1}{3}$.

The discussion is essentially identical to that in the first case and hence is omitted. 
3. $\left(q_{A}, q_{B}\right) \in R_{3}: q_{A}<\frac{k}{4-3 k}$ and $q_{B} \geq \frac{3-2 k}{6(1-k)}$.

When

$$
x=\frac{3-2 k+\sqrt{(3-2 k)^{2}-12(4-3 k)(1-k) q_{A}}}{2(4-3 k)} \quad \text { and } \quad y=1,
$$

$q_{A}<\frac{k}{4-3 k}$ implies that $x \in\left(\frac{3(1-k)}{4-3 k}, \frac{3-2 k}{4-3 k}\right)$. It then follows that

$$
\begin{aligned}
& 1-2 k e_{j}\left(p^{1}, \emptyset, \emptyset\right)=1-k(x+1)<2(1-k)(2 x-1), \quad \text { and } \\
& 1-2 k e_{j}\left(p^{1}, \emptyset, A\right)=1-k x>2(1-k)(2 x-1) .
\end{aligned}
$$

By Lemma 2, hence, $\left(d_{i}^{1}, d_{j}^{1}\right)=(\emptyset, \emptyset)$ is followed by a $B$-corner equilibrium and $\left(d_{i}^{1}, d_{j}^{1}\right)=(\emptyset, A)$ is followed by an interior equilibrium in period 2. Furthermore, the expected price of $B$ in period 2 equals

$$
\begin{aligned}
E\left[p_{B}^{2 *}\left(d^{1}\right)\right] & =x p_{B}^{2 *}(\emptyset, A)+(1-x) p_{B}^{2 *}(\emptyset, \emptyset) \\
& =x \frac{-1+k x+2(1-k)(2-x)}{3}+(1-x)\{-1+k(1+x)+2(1-k) x\} \\
& =\frac{-3(1-k)+2 x(6-5 k)-2 x^{2}(4-3 k)}{3} \\
& =-\frac{2}{3}(4-3 k)\left\{x-\frac{6-5 k}{2(4-3 k)}\right\}^{2}+\frac{(6-5 k)^{2}}{6(4-3 k)}-(1-k) \\
& \leq \frac{(6-5 k)^{2}}{6(4-3 k)}-(1-k) .
\end{aligned}
$$

On the other hand, since $q_{B} \geq \frac{3-2 k}{6(1-k)} \Leftrightarrow p_{B}^{1} \geq \frac{3-2 k}{6}$, the expected price of $B$ in period 2 is lower than $p_{B}^{1}$ if

$$
\frac{(6-5 k)^{2}}{6(4-3 k)}-(1-k)<\frac{3-2 k}{6} \Leftrightarrow k \in(0,1) .
$$

Therefore, any type $s_{i}$ is better off waiting and choosing $B$ in period 2 after any $d_{j}^{1}$ than choosing $B$ in period 1 . Furthermore, since $x<\frac{3-2 k}{4-3 k}$,

$$
x<\frac{1-k(x+1)}{6(1-k)}+\frac{x+1}{3}<\frac{1-k x}{6(1-k)}+\frac{x+1}{3} \leq 1,
$$

where the two quantities in the middle are the critical types who are indifferent between $A$ and $B$ in period 2 after $d_{j}^{1}=\emptyset$ and $d_{j}^{1}=A$, respectively. It follows that there are the following four possibilities to consider.

- Type $s_{i}$ chooses $A$ in period 1: $s_{i}<x$. 
- Type $s_{i}$ waits in period 1 and then in period 2 chooses $A$ after $d_{j}^{1}=A$ or $d_{j}^{1}=\emptyset: s_{i} \in\left(x, \frac{1-k(1+x)}{6(1-k)}+\frac{x+1}{3}\right)$.

By construction, choosing $A$ in period 1 yields the same payoff as waiting and then choosing $A$ after any $d_{j}^{1}$. On the other hand, waiting in period 1 is at least as good as waiting and then choosing $B$ after any $d_{j}^{1}$, and the latter dominates choosing $B$ in period 1 by the above discussion. Hence, choosing $A$ and waiting are both optimal in period 1 for any type in the above two cases.

- Type $s_{i}$ waits in period 1 and then in period 2 chooses $A$ after $d_{j}^{1}=A$ and chooses $B$ after $d_{j}^{1}=\emptyset: s_{i} \in\left(\frac{1-k(1+x)}{6(1-k)}+\frac{x+1}{3}, \frac{1-k x}{6(1-k)}+\frac{x+1}{3}\right)$.

- Type $s_{i}$ waits in period 1 and then in period 2 chooses $B$ after $d_{j}^{1}=A$ or $\emptyset$ : $s_{i}>\frac{1-k x}{6(1-k)}+\frac{x+1}{3}$.

In these two cases, type $s_{i}$ prefers choosing $B$ in period 2 after some $d_{j}^{1}$ to choosing $A$ after any $d_{j}^{1}$. By construction, waiting and then choosing $A$ after any $d_{j}^{1}$ yields exactly the same payoff as choosing $A$ in period 1 . Hence, he prefers waiting to choosing $A$ in period 1. On the other hand, waiting in period 1 is at least as good as waiting and then choosing $B$ after any $d_{j}^{1}$, and the latter dominates choosing $B$ in period 1 by the above discussion. Hence, waiting is optimal for type $s_{i}$.

4. $\left(q_{A}, q_{B}\right) \in R_{4}$ : This case is similar to when $\left(q_{A}, q_{B}\right) \in R_{2}$.

5. $\left(q_{A}, q_{B}\right) \in R_{5}$ : This case is similar to when $\left(q_{A}, q_{B}\right) \in R_{3}$.

6. $\left(q_{A}, q_{B}\right) \in R_{6}: \min \left\{q_{A}, q_{B}\right\}>1$.

Every type waits since

$$
x=0 \quad \text { and } \quad y=1 \text {. }
$$

The equilibrium price pair in period 2 then equals $\left(p_{A}^{2 *}(\emptyset, \emptyset), p_{B}^{2 *}(\emptyset, \emptyset)\right)=(1-k, 1-k)$. It follows that waiting is optimal since it yields

$$
\max \left\{E\left[v_{i} \mid s_{i}\right]-(1-k), E\left[w_{i} \mid s_{i}\right]-(1-k)\right\},
$$

whereas choosing $A$ or $B$ in period 1 yields at most

$$
\max \left\{E\left[v_{i} \mid s_{i}\right]-(1-k) q_{A}, E\left[v_{i} \mid s_{i}\right]-(1-k) q_{B}\right\} .
$$

7. $\left(q_{A}, q_{B}\right) \in R_{7}: \max \left\{q_{A}, q_{B}\right\}<\frac{3-2 k}{6(1-k)}$. 
No type waits since

$$
x=y=\frac{1-q_{A}+q_{B}}{2} .
$$

By construction, the conditional belief $P_{i}(\cdot \mid h)$ of $s_{i}$ given $h \in H_{i}$ is the uniform distribution over $[0,1]$. Hence, any buyer who waits will face the price pair (1$k, 1-k)$ in period 2 .

Consider any type $s_{i}<x$. He prefers $A$ to $B$ if forced to choose between them in period 1. If he waits and chooses $A$ after any $d_{j}^{1}$, then his payoff from waiting is dominated because he faces a higher price of $A$ in period 2. If he waits and then chooses $A$ after $d_{j}^{1}=A$ and $B$ after $d_{j}^{1}=B$, then his payoff is given by

$$
\begin{aligned}
& x E\left[v_{i} \mid s_{i}, p^{1}, d_{j}^{1}=A\right]+(1-x) E\left[w_{i} \mid s_{i}, p^{1}, d_{j}^{1}=B\right]-(1-k) \\
& x\left\{u+1-(1-k) s_{i}-k \frac{x}{2}\right\}+(1-x)\left\{u+(1-k) s_{i}+k \frac{1+x}{2}\right\}-(1-k) \\
& =u+x-(1-k)+(1-k) s_{i}(1-2 x)+\frac{k}{2}\left(1-2 x^{2}\right) .
\end{aligned}
$$

On the other hand, choosing $A$ in period 1 yields

$$
E\left[v_{i} \mid s_{i}\right]-(1-k) q_{A}=u+1-(1-k) s_{i}-\frac{k}{2}-(1-k) q_{A} .
$$

Choosing $A$ in period 1 is hence optimal if

$$
\begin{aligned}
& u+x-(1-k)+(1-k) s_{i}(1-2 x)+\frac{k}{2}\left(1-2 x^{2}\right) \\
& \leq u+1-(1-k) s_{i}-\frac{k}{2}-(1-k) q_{A},
\end{aligned}
$$

or equivalently,

$$
(1-k) q_{A} \leq 2-x-k-2(1-x)(1-k) s_{i}-k\left(1-x^{2}\right) .
$$

Since $s_{i}<x$ and $q_{A}<\frac{3-2 k}{6(1-k)}$, this is in turn implied by

$$
\begin{aligned}
\frac{3-2 k}{6} & \leq 2(1-k)-(3-2 k) x+(2-k) x^{2} \\
& =(2-k)\left\{x-\frac{3-2 k}{2(2-k)}\right\}^{2}+2(1-k)-\frac{(3-2 k)^{2}}{4(2-k)} .
\end{aligned}
$$

We can verify that this inequality holds since $k<\frac{1}{2}$ and

$$
\frac{3-2 k}{6} \leq 2(1-k)-\frac{(3-2 k)^{2}}{4(2-k)} \quad \Leftrightarrow \quad(1-2 k)(9-4 k) \geq 0 .
$$

The symmetric argument proves that choosing $B$ in period 1 is optimal when $s_{i}>y$. 
Step 2. We now examine the optimality of the price $q$ in (26).

Since $\frac{3-2 k}{6(1-k)}<q<1$, the price pair $(q, q)$ belongs to the interior of region 1 in Figure 6. Hence, for any $\left(q_{A}, q_{B}\right)$ in the neighborhood of $(q, q)$, any history $h=\left(p^{1}, d^{1}\right)$ is followed by an interior equilibrium of Lemma 2 . It follows that the equilibrium price must satisfy the first-order condition $\frac{\partial \hat{\Pi}_{A}}{\partial q_{A}}\left(q_{A}, q_{B}\right)=0$. Partially differentiating $\hat{\Pi}_{A}$ with respect to $q_{A}$, we obtain

$$
\begin{aligned}
\frac{\partial \hat{\Pi}_{A}}{\partial q_{A}}\left(q_{A}, q_{B}\right) & =(1-k)\left(\frac{1}{2}-\frac{2 q_{A}-q_{B}}{2}\right) \\
& +\frac{k^{2}}{18(1-k)}\left[-\frac{1}{2}\left(\frac{1}{2}+\frac{2 q_{A}-q_{B}}{2}\right)\left(1-\frac{q_{A}+q_{B}}{2}\right)\right. \\
& +\left(\frac{1}{2}+\frac{2 q_{B}-q_{A}}{2}\right)\left(1-\frac{q_{A}+q_{B}}{2}\right) \\
& \left.-\frac{1}{2}\left(\frac{1}{2}+\frac{2 q_{B}-q_{A}}{2}\right)\left(\frac{1}{2}+\frac{2 q_{A}-q_{B}}{2}\right)\right] .
\end{aligned}
$$

If $(q, q)$ is the symmetric period 1 price profile in equilibrium, then $\frac{\partial \hat{\Pi}_{A}}{\partial q_{A}}(q, q)=0$ must hold. Substitution of $q_{A}=q_{B}=q$ into (29) yields upon simplification

$$
\frac{1}{2}(1-k)+\frac{k^{2}}{144(1-k)}-\frac{1-k}{2} q+\frac{k^{2}}{72(1-k)}\left[-q-\frac{3}{2} q^{2}\right]=0,
$$

or equivalently,

$$
3 k^{2} q^{2}+2\left\{36(1-k)^{2}+k^{2}\right\} q-\left\{72(1-k)^{2}+k^{2}\right\}=0 .
$$

The non-negative solution to this quadratic equation is given by (26).

We now show that any $q \in(0,1)$ satisfying $(30)$ is a global maximizer: $\hat{\Pi}_{A}(q, q)>$ $\hat{\Pi}_{A}\left(q_{A}, q\right)$ for any $q_{A} \neq q$.

a) $\left(q_{A}, q\right) \in R_{1}: 2 q_{A}-q \leq 1$ and $2 q-q_{A} \leq 1$.

The second-order derivative of $\hat{\Pi}\left(q_{A}, q_{B}\right)$ with respect to $q_{A}$ is given by

$$
\frac{\partial^{2} \hat{\Pi}_{A}}{\partial q_{A}^{2}}\left(q_{A}, q_{B}\right)=-1+\frac{k^{2}}{72(1-k)^{2}}\left(-5-3 q_{B}+6 q_{A}\right),
$$

which is $<0$ when $q_{A} \leq \frac{q_{B}+1}{2}$. It follows that $\frac{\partial \hat{\Pi}_{A}}{\partial q_{A}}\left(q_{A}, q_{B}\right)$ is strictly decreasing for any such $q_{A}$. This in turn implies that $q_{A}=q$ maximizes $\hat{\Pi}_{A}\left(q_{A}, q\right)$ over $q_{A} \in$ $\left[2 q-1, \frac{q+1}{2}\right]$. 
b) $\left(q_{A}, q\right) \in R_{2}: \frac{k}{4-3 k} \leq q_{A} \leq 2 q-1$.

Since $y=1$, we have either $d_{j}^{1}=A$ or $\emptyset$, and both $d^{1}=(\emptyset, A)$ and $(\emptyset, \emptyset)$ are followed by an interior equilibrium in period 2 . Since

$$
\begin{aligned}
p_{A}^{1} & =x p_{A}^{2 *}(\emptyset, A)+(1-x) p_{A}^{2 *}(\emptyset, \emptyset) \\
& =x \frac{1-k x+2(1-k)(1-2 x)}{3}+(1-x) \frac{1-k(x+1)+2(1-k)(1-2 x)}{3} \\
& =(1-k) \frac{3-4 x}{3}
\end{aligned}
$$

we have

$$
q_{A}=\frac{3-4 x}{3},
$$

and $q_{A} \in\left[\frac{k}{4-3 k}, 2 q-1\right] \Leftrightarrow x \in\left[\frac{3(1-k)}{4-3 k}\right] . \hat{\Pi}_{A}$ can be expressed in terms of $x$ as:

$$
\begin{aligned}
& \hat{\Pi}_{A}\left(q_{A}, q_{B}\right) \\
& =(1-k) q_{A} x+(1-x)\left\{x \pi_{A}^{2 *}(\emptyset, A)+(1-x) \pi_{A}^{2 *}(\emptyset, \emptyset)\right\} \\
& =(1-k) \frac{3-4 x}{3} x \\
& +\frac{1}{18(1-k)}[\{1+2(1-k)(1-2 x)\}\{1-2 k+2(1-k)(1-2 x)\} \\
& \left.\quad+k^{2}+k^{2} x(1-x)\right]
\end{aligned}
$$

We can verify that $\frac{\partial \hat{\Pi}_{A}}{\partial x}<0$ for $x \in$ so that $\frac{\partial \hat{\Pi}_{A}}{\partial q_{A}}>0$ for $q_{A} \in$. It is hence maximized over this region when $q_{A}=2 q-1$. Since $\hat{\Pi}_{A}$ is continuous at $q_{A}=2 q-1$, we have $\hat{\Pi}_{A}(2 q-1, q)<\hat{\Pi}_{A}(q, q)$ from case 1 above.

c) $\left(q_{A}, q\right) \in R_{3}: q_{A}<\frac{k}{4-3 k}$.

Since $y=1$ again, we have either $d_{j}^{1}=A$ or $\emptyset . d^{1}=(\emptyset, A)$ is followed by an interior equilibrium in period 2, while $d^{1}=(\emptyset, \emptyset)$ is followed a $B$-corner equilibrium in period 2. Since

$$
\begin{aligned}
p_{A}^{1} & =x p_{A}^{2 *}(\emptyset, A)+(1-x) p_{A}^{2 *}(\emptyset, \emptyset) \\
& =x \frac{1-k x+2(1-k)(1-2 x)}{3}+(1-x) 0 \\
& =x \frac{1-k x+2(1-k)(1-2 x)}{3},
\end{aligned}
$$

we have

$$
q_{A}=x \frac{1-k x+2(1-k)(1-2 x)}{3(1-k)},
$$


and $q_{A} \in\left[0, \frac{k}{4-3 k}\right) \Leftrightarrow x \in\left[\frac{3(1-k)}{4-3 k}, \frac{3-2 k}{4-3 k}\right] . \hat{\Pi}_{A}$ can be expressed in terms of $x$ as

$$
\begin{aligned}
\hat{\Pi}_{A}\left(q_{A}, q_{B}\right) & =(1-k) q_{A} x+(1-x) x \pi_{A}^{2 *}(\emptyset, A) \\
& =\frac{x^{2}}{3}\{1-k x+2(1-k)(1-2 x)\} \\
& +\frac{x}{18(1-k)}\{1-k x+2(1-k)(1-2 x)\}^{2} \\
& =\frac{x}{18(1-k)}\{1-k x+2(1-k)(1-2 x)\} \\
& \quad \times\{1-k x+2(1-k)(1+x)\} .
\end{aligned}
$$

After some algebra, we see that

$$
\frac{\partial^{2} \hat{\Pi}_{A}}{\partial x^{2}}=\frac{3 x\{k+(1-k)(9 k-8)\}-6+4 k}{9(1-k)}<0,
$$

and

$$
\left.\frac{\partial \hat{\Pi}_{A}}{\partial x}\right|_{x=\frac{3(1-k)}{4-3 k}}<0 .
$$

These inequalities together imply that $\frac{\partial \hat{\Pi}_{A}}{\partial x}<0$ over $x \in\left(\frac{3(1-k)}{4-3 k}, \frac{3-2 k}{4-3 k}\right)$, and hence that $\hat{\Pi}_{A}$ is maximized when $x=\frac{3(1-k)}{4-3 k}$. Equivalently, $\hat{\Pi}_{A}\left(q_{A}, q\right)$ is maximized when $q_{A}=\frac{k}{4-3 k}$ over $q_{A} \in\left[0, \frac{k}{4-3 k}\right]$. Since $\hat{\Pi}_{A}\left(q_{A}, q\right)$ is continuous at $q_{A}=\frac{k}{4-3 k}$, we conclude that $\hat{\Pi}_{A}\left(\frac{k}{4-3 k}, q\right)<\hat{\Pi}_{A}(q, q)$ from the above two cases.

d) $\left(q_{A}, q\right) \in R_{4}: q_{A} \geq \frac{q+1}{2}$.

Since $x=0$, we have either $d_{j}^{1}=B$ or $\emptyset$, and both $d^{1}=(\emptyset, B)$ and $(\emptyset, \emptyset)$ are followed by an interior equilibrium in period 2. Firm $A$ 's payoff over two periods then equals:

$$
\begin{aligned}
& \hat{\Pi}_{A}\left(q_{A}, q_{B}\right) \\
& =y\left\{y \pi_{A}^{2 *}(\emptyset, \emptyset)+(1-y) \pi_{A}^{2 *}(\emptyset, B)\right\} \\
& =\frac{1}{18(1-k)}\left[y\{1-k y+2(1-k) y\}^{2}\right. \\
& \left.\quad+(1-y)\{1-k(1+y)+2(1-k) y\}^{2}\right] .
\end{aligned}
$$

By Lemma 4, we have

$$
\begin{aligned}
p_{B}^{1} & =y p_{B}^{2 *}(\emptyset, \emptyset)+(1-y) p_{B}^{2 *}(\emptyset, B) \\
& =y \frac{-1+k y+2(1-k)(2 y-1)}{3}+(1-y) \frac{-1+k(1+y)+2(1-k)(2 y-1)}{3} \\
& =\frac{-1+k+2(1-k)(2 y-1)}{3},
\end{aligned}
$$


or equivalently, $y=\frac{3\left(q_{B}+1\right)}{4}$. Hence $y$ is independent of $q_{A}$, and so is $\hat{\Pi}_{A}$. It follows that $\hat{\Pi}_{A}\left(q_{A}, q\right)=\hat{\Pi}_{A}\left(\frac{q+1}{2}, q\right)$. Since $\hat{\Pi}_{A}(\cdot, q)$ is continuous at $q_{A}=\frac{q+1}{2}$, the analysis in the first case implies that $\hat{\Pi}_{A}(q, q)>\hat{\Pi}_{A}\left(q_{A}, q\right)$ for any $a_{A} \geq \frac{q+1}{2}$.

This completes the proof.

\section{References}

[1] Masaki Aoyagi, 2010, "Optimal Sales Schemes against Interdependent Buyers," American Economic Journal: Microeconomics, 2(1), 150-182.

[2] Abhijit Banerjee, 1992, "A Simple Model of Herd Behavior," Quarterly Journal of Economics, 107, 797-817.

[3] David Besanko, and Wayne L. Winston, 1990, "Optimal Price Skimming by a Monopolist Facing Rational Consumers," Management Science, 36(5), 555-567.

[4] Manaswini Bhalla, 2012, "Dynamic Pricing under Social Learning with Strategic Consumers," Working Paper.

[5] Manaswini Bhalla, 2013, "Waterfall versus Sprinkler Product Launch Strategy: Influencing the Herd," Journal of Industrial Economics, 61, 138-165.

[6] Sushil Bikhchandani, David Hirshleifer, and Ivo Welch, 1992, "A Theory of Fads, Fashion, Custom and Cultural Change as Informational Cascades," Journal of Political Economy, 100, 992-1026.

[7] Subir Bose, Gerhart Orosel, Marco Ottaviani, and Lise Vesterlund, 2006, "Dynamic Monopoly Pricing and Herding," RAND Journal of Economics, 37, 910-928.

[8] Subir Bose, Gerhart Orosel, Marco Ottaviani, and Lise Vesterlund, 2008, "Monopoly Pricing in the Binary Herding Model," Economic Theory, 37, 203-41.

[9] Christophe Chamley, 2004, Rational Herds: Economic Models of Social Learning, Cambridge University Press: Cambridge.

[10] Christophe Chamley and Douglas Gale, 1994, "Information Revelation and Strategic Delay in a Model of Investment," Econometrica, 62, 1065-1085.

[11] Yuxin Chen, and Z. John Zhang, 2009, "Dynamic Targeted Pricing with Strategic Consumers," International Journal of Industrial Organization, 27, 43-50. 
[12] Jérémy Gallien, 2006, "Dynamic Mechanism Design for Online Commerce," Operations Research, 54(2) 291-310.

[13] Jochen Gönsch, Robert Klein, Michael Neugebauer, and Claudius Steinhardt, 2012, "Dynamic Pricing with Strategic Consumers," Journal of Business Economics, 83(5), 505-549.

[14] Faruk Gul and Russell J. Lundholm, 1995, "Endogenous Timing and the Clustering of Agents' Decisions," Journal of Political Economy, 103(5), 1039-1066.

[15] Hikmet Gunay, 2008, "The Role of Externalities and Information Aggregation in Market Collapse," Economic Theory, 35, 367-379.

[16] Hikmet Gunay, 2008, "Information Aggregation under Strategic Delay," Economics Bulletin, 12(23), 1-8.

[17] Hikmet Gunay, 2013, "Waiting for Signalling Quality," Southern Economic Journal, forthcoming.

[18] Philip Jehiel and Benny Moldovanu, 2001, "Efficient Design with Interdependent Valuations," Econometrica, 69(5), 1237-1259.

[19] Yuri Levin, Jeff McGill, and Mikhail Nediak, 2009, "Dynamic Pricing in the Presence of Strategic Consumers and Oligopolistic Competition," Management Science, $55(1), 32-46$.

[20] Qian Liu, and Dan Zhang, 2013, "Dynamic Pricing Competition with Strategic Customers under Vertical Product Differentiation," Management Science, 59(1), 84-101.

[21] Vincent Mak, Amnon Rapoport, and Eyran J. Gisches, 2012, "Competitive Dynamic Pricing with Alternating Offers: Theory and Experiment," Games and Economic Behavior, 75, 250-264.

[22] Daniel Sgroi, 2002, "Optimizing Information in the Herd: Guinea Pigs, Profits and Welfare," Games and Economic Behavior, 39, 137-166.

[23] Robert J. Weber, 1981, "Multiple-Object Auctions," Discussion Paper 496, Northwestern University.

[24] Man Yu, Roman Kapuscinski, and Hyun-Soo Ahn, 2011, "Advance Selling with Interdependent Customer Valuations," working paper. 